Revue d'histoire de l'Amérique française

\title{
Parenté et stratification sociale dans une paroisse rurale de la vallée du Saint-Laurent au milieu du XIX ${ }^{\mathrm{e}}$ siècle1
}

\section{Christian Dessureault}

Volume 54, numéro 3, hiver 2001

URI : https://id.erudit.org/iderudit/005397ar

DOI : https://doi.org/10.7202/005397ar

Aller au sommaire du numéro

\section{Éditeur(s)}

Institut d'histoire de l'Amérique française

\section{ISSN}

0035-2357 (imprimé)

1492-1383 (numérique)

Découvrir la revue

\section{Citer cet article}

Dessureault, C. (2001). Parenté et stratification sociale dans une paroisse rurale de la vallée du Saint-Laurent au milieu du XIX ${ }^{\mathrm{e}}$ siècle1. Revue d'histoire de l'Amérique française, 54(3), 411-447. https://doi.org/10.7202/005397ar
Résumé de l'article

Les réseaux et les rapports de parenté représentent des vecteurs importants des recherches sur la société rurale du Québec. Cependant, nous n'avons encore aucune étude qui évalue de manière systématique l'importance des liens de parenté dans une paroisse rurale à un moment spécifique de l'histoire. Nous ne connaissons pas, de manière précise, la densité des liens familiaux entre les divers ménages d'une même paroisse, ni surtout le degré de variabilité de ces liens de parenté selon les familles, selon le genre et selon les groupes sociaux.Cette recherche vise à présenter une première mesure concernant l'étendue et les types des liens de parenté entre tous les chefs de ménage d'une vieille paroisse rurale de la vallée du Saint-Laurent en 1861: Saint-Antoine-de-Lavaltrie. L'un des principaux objectifs de cet article est de vérifier l'étendue, la sélectivité sociale et la différenciation selon le genre des réseaux de parenté dans un territoire donné où se vivent, au quotidien, les relations sociales. Cet article aide à mieux circonscrire le poids différencié des rapports de parenté dans les divers groupes au sein des communautés rurales. Cette recherche permet aussi de corroborer ou de nuancer, dans un contexte historique précis, certains constats de la sociologie, de l'anthropologie et de l'histoire sociale concernant l'importance et la structure des réseaux de parenté.
Tous droits réservés @ Institut d'histoire de l'Amérique française, 2001
Ce document est protégé par la loi sur le droit d'auteur. L'utilisation des services d'Érudit (y compris la reproduction) est assujettie à sa politique d'utilisation que vous pouvez consulter en ligne.

https://apropos.erudit.org/fr/usagers/politique-dutilisation/ 


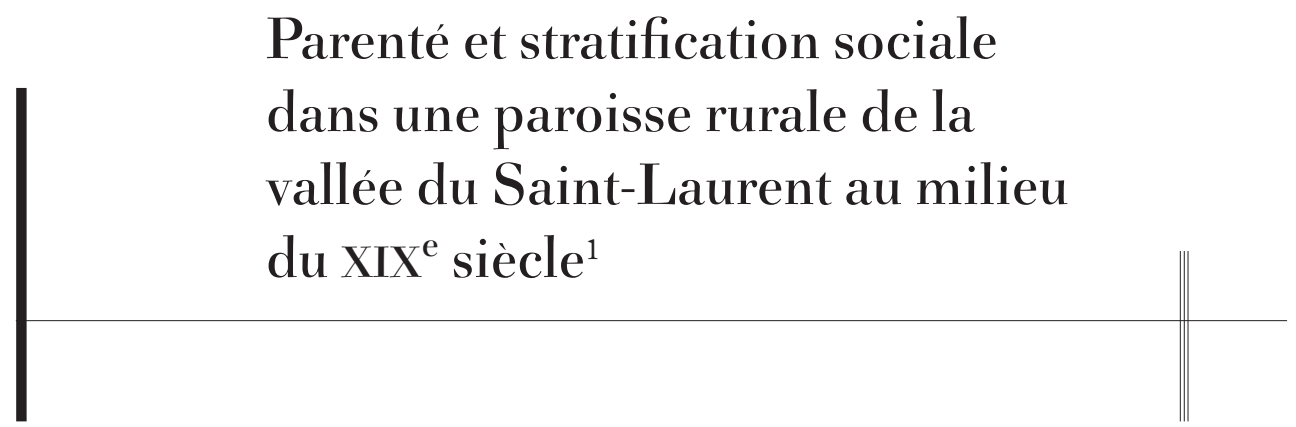

CHRISTIAN DESSUREAULT

Département d'histoire

Université de Montréal

RÉSUMÉ - Les réseaux et les rapports de parenté représentent des vecteurs importants des recherches sur la société rurale du Québec. Cependant, nous n'avons encore aucune étude qui évalue de manière systématique l'importance des liens de parenté dans une paroisse rurale à un moment spécifique de l'histoire. Nous ne connaissons pas, de manière précise, la densité des liens familiaux entre les divers ménages d'une même paroisse, ni surtout le degré de variabilité de ces liens de parenté selon les familles, selon le genre et selon les groupes sociaux.

Cette recherche vise à présenter une première mesure concernant l'étendue et les types des liens de parenté entre tous les chefs de ménage d'une vieille paroisse rurale de la vallée du Saint-Laurent en I86I : Saint-Antoine-de-Lavaltrie. L'un des principaux objectifs de cet article est de vérifier l'étendue, la sélectivité sociale et la différenciation selon le genre des réseaux de parenté dans un territoire donné où se vivent, au quotidien, les relations sociales. Cet article aide à mieux circonscrire le poids différencié des rapports de parenté dans les divers groupes au sein des communautés rurales. Cette recherche permet aussi de corroborer ou de nuancer, dans un contexte historique précis, certains constats de la sociologie, de l'anthropologie et de l'histoire sociale concernant l'importance et la structure des réseaux de parenté.

ABSTRACT - Family networks and relationship constitute important vectors of research on Quebec rural society. However, there is no study, as yet, that evaluates systematically the importance of family ties in a rural parish at a specific time of history. We do not know with precision the density of relationship among the various households from the same

1. Nous tenons à remercier le Fonds FCAR, de même que Diane Gervais et Thomas Wien qui ont commenté une première version de ce texte. 
parish, nor moreover the variability degree of kinship according to family, to gender and to social groups.

The present article offers a first evaluation of the size and the types of relationship within all the householders from an old St. Lawrence valley rural parish in 1861: Saint-Antoine-deLavaltrie. One of the main goals of this article is to verify the extent, the social selectivity and the differenciation according to gender of family ties in a given territory where social relations are lived on a daily basis. This article contributes to a better understanding of the differenciated weight of family kinship in the various groups constituting rural communities. It also helps to corroborate or to qualify within a precise historical context some statements made by sociologists, anthropologists and social historians concerning the importance and the structure of family networks.

D ANS UN ARTICLE portant sur les fabriques paroissiales, publié l'an dernier dans la revue The Canadian Historical Review ${ }^{2}$, nous avons consacré une section de cette étude à vérifier la densité des réseaux familiaux parmi les marguilliers élus des paroisses étudiées. Nous avons alors constaté l'existence de nombreux liens de parenté entre ces élites institutionnelles locales et interprété la présence de réseaux familiaux comme l'un des indices de la concentration et de la sélectivité de ces charges publiques. Mais si la plupart des ménages des paroisses rurales du Québec de cette époque étaient assez généralement apparentés les uns aux autres, l'ampleur de ces réseaux de parenté ne serait pas vraiment déterminante. Néanmoins, cette conviction d’un Québec rural aussi "tricoté serré» appartient, selon nous, davantage au cliché ou au mythe populaire qu'à un constat fondé sur des connaissances scientifiques. Dans un article consacré aux origines de la population saguenayenne, Gérard Bouchard et Lise Bergeron ont bien montré les méprises de la mémoire collective sur ce type de questions ${ }^{3}$. Néanmoins, nous devons reconnaître l'absence, dans la production scientifique québécoise, de données pertinentes permettant de mesurer adéquatement la densité relative des liens de parenté dans des territoires donnés, des paroisses rurales ou des quartiers urbains, à des périodes variables de l’histoire. Pourtant, la parenté n’est pas un nouvel

2. C. Dessureault et C. Hudon, "Conflits sociaux et élites locales au début du $\mathrm{XIx}^{\mathrm{e}}$ siècle. Le clergé, les notables, la paysannerie et contrôle de la fabrique », The Canadian Historical Review, 80,3 (septembre 1999) : 413-439.

3. Ces derniers ont entre autres souligné que, contrairement aux croyances populaires, la fréquence des mariages consanguins n’était pas plus élevée au Saguenay-Lac-Saint-Jean que dans les autres régions du Québec et que le bassin génétique de la population de cette région était assez diversifié. G. Bouchard et L. Bergeron, «Aux origines d'une population régionale : mythes et réalités démographiques et sociales ", Revue d'histoire de l'Amérique française, 42,3 (hiver 1989): 389-410. 
objet d'étude en histoire sociale. L'emploi des concepts de rapports de parenté et de réseaux de parenté, empruntés à l'anthropologie et à l'ethnologie, ont influencé plusieurs champs de la recherche historique : les alliances matrimoniales, la transmission des patrimoines, les migrations et la formation de la classe ouvrière.

Les réseaux de parenté et les rapports de parenté représentent des vecteurs importants de l'histoire sociale et, plus spécifiquement, des recherches actuelles sur la société rurale du Québec. Nous connaissons désormais un peu mieux le poids des réseaux de parenté sur certains aspects de la vie des familles et des groupes sociaux. Nous comprenons également un peu mieux l'importance des rapports de parenté dans le fonctionnement social. Cependant, nous n'avons encore aucune étude qui évalue de manière systématique l'importance des liens de parenté dans une paroisse rurale, dans un bourg ou dans un quartier urbain à un moment spécifique de l'histoire. Nous ne connaissons donc pas, de manière précise, la densité des liens familiaux entre les divers résidants d'une même paroisse ou d'un même quartier urbain, ni surtout le degré de variabilité de ces liens de parenté selon les familles, selon le genre et selon les groupes sociaux.

Cette recherche vise donc à présenter une première mesure concernant l'étendue et les types des liens de parenté entre tous les chefs de ménage d'une vieille paroisse rurale de la vallée du Saint-Laurent au milieu du xIx $^{\mathrm{e}}$ siècle : Saint-Antoine-de-Lavaltrie. L'approche et la méthode peuvent éventuellement servir à effectuer des recherches comparatives, dans le temps et dans l'espace, sur des paroisses de peuplement récent ou des quartiers urbains.

L'un de nos principaux objectifs est de vérifier l'étendue, la sélectivité sociale et la différenciation selon le genre des réseaux de parenté dans un territoire donné où se vivent, au quotidien, les relations sociales. Cette étude contribuera certainement à mieux circonscrire la portée historiquement variable des rapports de parenté dans l'organisation sociale, dans l'univers de la sociabilité quotidienne, voire dans la stratification des communautés rurales. Cette recherche aidera également à étayer ou à nuancer, dans un contexte historique précis, certains constats de la sociologie, de l'anthropologie et de l'histoire sociale concernant l'importance et la structure des réseaux de parenté.

Nous voulons entre autres mesurer l'étendue, la nature et les limites sociales des liens de parenté selon le statut socioprofessionnel des chefs de ménage : journaliers, artisans, cultivateurs, notables. Nous pourrons ainsi, 
de manière indirecte, appréhender la distance ou la proximité des divers groupes professionnels dans l'espace social et paroissial. Dans la même perspective, nous essayerons aussi de déterminer, par le biais de cette même analyse des réseaux familiaux, certains impacts possibles de la différenciation économique au sein du groupe paysan. Nous aborderons aussi l'étude de la structure des ménages qui, dans la littérature scientifique et dans la société étudiée, est difficilement dissociable de la question de la place de la parenté dans le tissu social des communautés.

\section{UN BILAN DE LA QUESTION DE LA PARENTÉ AU QUÉBEC}

Dans un premier temps, nous voulons présenter un bref bilan de la question de la parenté dans la littérature scientifique au Québec d'une part, pour mieux situer l'apport spécifique de cette étude dans le renouvellement de cet objet commun à plusieurs disciplines des sciences sociales et, d'autre part, pour en dégager un certain nombre d'acquis ou de réflexions ayant servi à étayer notre propre démarche.

Ce champ de recherche est relativement récent chez les historiens et encore peu fréquenté des disciples de Clio. Pourtant, la dynamique des rapports de parenté est déjà présente en filigrane, dès le tournant du siècle, dans les recherches menées par le sociologue Léon Gérin sur la société rurale canadienne-française $e^{4}$. Toutefois, les sociologues de l'École de Chicago sont les premiers chercheurs à mettre l'accent, dès la fin des années 1930, sur la fonction déterminante des rapports de parenté dans cette société rurale qu'ils définissent par ailleurs comme l'une des variantes de la Folk Society ${ }^{5}$. Ces chercheurs soulignent la place de la famille comme unité sociale fondamentale et, surtout, ils insistent sur l'insertion de la famille dans un ensemble plus large de relations sociales, fondées principalement sur la parenté.

Dans les années 1950, les sociologues québécois questionnent à leur tour le système de parenté dans la société canadienne-française tantôt pour réfuter, tantôt pour nuancer, le modèle de la Folk Society. Dans une étude sur le milieu urbain montréalais au $\mathrm{xx}^{\mathrm{e}}$ siècle, Philippe Garigue reconnaît l'importance de la parenté en insistant toutefois sur la primauté

4. L. Gérin, Le type économique et social des Canadiens. Milieux agricoles et tradition française (Montréal, Éditions de l'Association canadienne-française, science sociale, 1938). Cet ouvrage rassemble cinq monographies de familles rurales de divers milieux réalisées par Gérin entre 1880 et 1920 .

5. H. Miner, St.Denis. A French-Canadian Parish (Chicago, The University of Chicago Press, 1939); E. C. Hugues, French Canada in Transition (Chicago, University of Chicago Press, 1943). 
de la famille conjugale. Il met aussi l'accent sur la fonction différenciée et l'intensité variable des relations entre, d'une part, une parenté proche se limitant essentiellement aux ascendants et aux descendants directs d'un individu, de même qu'à ses frères, ses sœurs et leurs conjoints et, d'autre part, une parenté éloignée, c'est-à-dire tous les autres parents ${ }^{6}$. La parenté proche commande des liens de solidarité assortis d'obligations dans la vie quotidienne, tandis que la parenté éloignée suscite plutôt des relations de convenance, liées aux circonstances et aux événements plus exceptionnels de la vie sociale. De plus, selon Garigue, les individus peuvent exercer assez aisément des choix préférentiels dans leurs relations au sein de la parenté éloignée. Malgré l’importance symbolique de la lignée, les rapports sont plus fréquents entre les parents d'une même génération «[...] et cela sans égard aux liens de consanguinité et d'affinité par alliance ${ }^{7}$ ». Par ailleurs, selon lui, les femmes exercent un rôle plus actif que les hommes pour assurer le fonctionnement et la persistance des réseaux de parenté. Par contre, pour Marcel Rioux, le système de parenté dans la société traditionnelle canadienne-française demeure plus proche du modèle de la Folk Society ${ }^{8}$. Selon lui, la souplesse des rapports de parenté décrits par Garigue est davantage le reflet de l'effritement du système traditionnel, lié à l'industrialisation et à l'urbanisation, que l'expression du modèle spécifique de la société rurale canadienne-française. Pour Rioux, le système de parenté représente dans cette société l'une des composantes fondamentales de l'organisation sociale et ce système de relations sociales est à la fois dense et étendu.

Dans les années subséquentes, les anthropologues québécois vont à leur tour explorer ce champ de recherche dans des travaux consacrés à l'étude de la société rurale. Ces derniers visent d'abord à mieux définir les structures de la parenté. Ils vérifient la fréquence de la consanguinité dans des communautés spécifiques et, surtout, ils déterminent les types privilégiés

6. P. Garigue, «Le système de parenté en milieu urbain canadien-français », dans M. Rioux et Y. Martin, La société canadienne-française. Études choisies (Montréal, Hurtubise HMH, 1971), 363376, version française d'un article paru dans American Anthropologist, 58,6 (1956) : 1090-1101; P. Garigue, Études sur le Canada français (Montréal, Université de Montréal, Faculté des sciences sociales, économiques et politiques, 1958).

7. P. Garigue, «Le système de parenté... », op. cit., 364.

8. M. Rioux, "La connaissance de la parenté et l'urbanisation du Canada français », dans M. Rioux et Y. Martin, La société canadienne-française. Études choisies (Montréal, Hurtubise HMH, 1971), 377-387, version française d'un article paru dans Contributions to Anthropology, 173 (1959): 1-11. 
de filiation dans les mariages consanguins ${ }^{9}$. Ces travaux sur la consanguinité vont, d'une part, conduire à l'essor subséquent, au Québec, d'un axe de recherche plus spécifique sur la génétique des populations ${ }^{10}$. L'étude des mariages consanguins et du système de parenté débouche, d'autre part, à la fin des années 1960, sur un essai d'interprétation des attitudes et des comportements politiques dans la société rurale ${ }^{11}$.

En 1970, Pierre Beaucage aborde la question de la parenté sous un nouvel angle en scrutant dans des communautés de pêcheurs les rapports entre les réseaux de parenté et le fonctionnement du système économique $^{12}$. Ce dernier fait alors ressortir l'organisation différenciée des réseaux de parenté des divers groupes familiaux selon la propriété des moyens de production et selon les rapports à la production. La prédominance des liens patrilinéaires dans les équipes de pêche à la morue est liée à la possession des équipements de production et au degré d'autonomie économique des familles de pêcheurs.

Plus tard, au début des années 1980, le sociologue Daniel Fournier relance l'étude de la consanguinité et des modèles de sociabilité en se penchant cette fois sur le milieu urbain, à Montréal, au début du xx ${ }^{e}$ siècle $^{13}$. En 1985, la direction de la revue Anthropologie et sociétés publie un numéro spécial sur la parenté au Québec dont certains articles apportent un nouvel éclairage sur les modèles différenciés de relation au sein de la parenté et sur la place des femmes dans le système de parenté ${ }^{14}$. À partir d'une étude des réseaux informels d'échange des biens de consommation et de service dans un village des environs de Québec, Andrée Roberge démontre la persistance de la parenté

9. J. Allard, Bois-Vert. Famille et parenté, mémoire de maitrise (anthropologie), Université de Montréal, 1967; L. Guyon, Évolution de la consanguinité à Bois-Vert, thèse de doctorat (anthropologie), Université de Montréal, 1968; P. Philippe, Structure de la consanguinité à l'Isle-aux-Coudres, thèse de doctorat (anthropologie), Université de Montréal, 1969; M. Levasseur, Saint-Rémi-deTriolet. Famille et parenté, thèse de doctorat (anthropologie), Université de Montréal, 1971.

10. La génétique des populations constitue par ailleurs depuis quelques années l'un des principaux champs de recherche de l'Institut de recherche sur les populations (IREP). G. Bouchard et M. De Braekeleer, dir., Histoire d'un génôme. Population et génétique dans l'Est du Québec (Québec, Presses de l’Université du Québec, 1991).

11. V. Lemieux, "Mariages consanguins et système de parenté dans l'île d'Orléans ", Recherches sociographiques, 7,3 (1966) : 349-359; V. Lemieux, Parenté et politique : l'organisation sociale dans l'Ŷle d'Orléans (Québec, Les Presses de l'Université Laval, 1971).

12. P. Beaucage, "Organisation économique et parenté à La Tabatière», Recherches sociographiques, 11, (1970) : 91-116.

13. D. Fournier, "Consanguinité et sociabilité dans la zone de Montréal au début du siècle», Recherches sociographiques, 24,3 (1983): 307-323.

14. "Parentés au Québec», Anthropologie et sociétés, 9,3 (1985). 
comme l'une des institutions fondamentales de l'organisation sociale du Québec contemporain et l'un des éléments structurants de la dynamique communautaire. Elle fait également ressortir la primauté des femmes dans le fonctionnement des réseaux d'échange et d'entraide au sein de la parenté et constate l'orientation matriarcale du système de parenté, du moins au niveau fonctionnel ${ }^{15}$. Par contre, dans ce même numéro spécial, Chantal Collard publie une étude sur le système de parenté dans une paroisse de Charlevoix, de 1880 à 1960, selon laquelle «à la discrimination des femmes via la patrilinéarité et la résidence virilocale s'oppose un autre trait du système de parenté qui est le parallélisme basé sur le sexe ${ }^{16}{ }^{1}$. Cette différenciation selon le genre des réseaux de parenté qui s'exprime entre autres par la proximité résidentielle des hommes et par la proximité cognitive des femmes repose d'abord, selon C. Collard, sur la division sexuelle des tâches. Dans un article subséquent, cette auteure réaffirme les traits spécifiques du système de parenté charlevoisien et explore brièvement l'impact de la différenciation sociale dans ce milieu relativement égalitaire. Elle souligne ainsi que «tant du point de vue de l'héritage que des choix matrimoniaux et de la résidence, le comportement des propriétaires terriens se distingue (du comportement) des non-propriétaires ${ }^{17}$ ». Dans un livre récent, C. Collard aborde de manière plus détaillée les multiples facettes des rapports de parenté dans cette même paroisse rurale de Charlevoix. Elle souligne plus particulièrement l'importance des représentations symboliques de la parenté et examine les divers impacts sociaux des rapports de parenté dans les domaines des alliances, de la transmission des biens et des rapports de genre ${ }^{18}$.

Les historiens québécois ont adopté plus tardivement les concepts de rapports de parenté et de réseaux de parenté, sous l'influence des recherches menées dans le domaine de l'histoire de la famille en Europe et aux États-Unis ${ }^{19}$. Les études sur le phénomène migratoire, tant l'émigration

15. A. Roberge, "Réseaux d’échange et parenté inconsciente», Anthropologie et sociétés, 9,3 (1985) : 5-31.

16. C. Collard, «Parenté et communauté à Rivière-Frémiotte, 1880-1960", Anthropologie et sociétés, 9,3 (1985) : 57-85.

17. C. Collard, «Idéologie et pratique de la parenté : de la classification des parents aux stratégies familiales", dans G. Bouchard et M. De Braekeleer, dir., Histoire d'un génôme. Population et génétique dans l'Est du Québec, op. cit., 121-144.

18. C. Collard, Une famille, un village, une nation. La parenté dans Charlevoix 1900-1960 (Montréal, Boréal, 1999).

19. Parmi les principaux ouvrages qui ont influencé l'évolution de l'histoire de la famille et plus spécifiquement l'étude des structures des ménages, des alliances matrimoniales, du cycle de vie familiale et des réseaux de parenté dans l’organisation sociale : P. Laslett, dir., Household and 
vers les États-Unis ${ }^{20}$, l'exode des ruraux vers la ville ${ }^{21}$ que les migrations internes dans la société rurale ${ }^{22}$, vont ainsi démontrer l'importance des réseaux de parenté dans le recrutement des migrants, dans leur sélection et leur mode d'implantation et d'insertion sociale dans leurs nouveaux milieux ${ }^{23}$. Par ailleurs, des études sur les artisans et sur la classe ouvrière montrent l'importance des réseaux de parenté et des solidarités familiales tantôt dans le fonctionnement et la capacité de résistance du mode de production artisanal $^{24}$, tantôt dans la formation initiale de la classe ouvrière et la création des premières organisations syndicales ${ }^{25}$. Plus récemment, nous avons constaté l'importance de réseaux familiaux dans la participation à certaines instances locales de pouvoir, dont la fabrique et la milice ${ }^{26}$.

the Family in Past Time (Cambridge, Cambridge University Press, 1972); T. K. Hareven, The Family Time and Industrial Time. The Relationship between the Family and Work in a New England Community (Cambridge, Cambridge University Press, 1982); M. Segalen, Quinze générations de Bas-Breton (Paris, Presses universitaires de France, 1985).

20. B. Ramirez et J. Lamarre, «Du Québec vers les États-Unis : l'étude des lieux d'origine», Revue d'histoire de l'Amérique française, 35,3 (hiver 1985) : 409-422; Y. Frenette, "La genèse d'une communauté canadienne-française en Nouvelle-Angleterre, Lewiston, Maine, 1800-1880", Communications historiques/Historical Papers (1989) : 75-99; B. Ramirez, Par monts et par vaux : migrants canadiens-français et italiens dans l'économie nord-atlantique, 1860-1914 (Montréal, Boréal, 1992).

21. F. Gagnon, «Parenté et migration : le cas des Canadiens français à Montréal entre 1845 et 1875 », Communications historiques/Historical Papers (1988) : 63-85 ; M. St-Hilaire, "La formation des populations urbaines au Québec : le cas du Saguenay aux $\mathrm{xIx}^{\mathrm{e}}$ et $\mathrm{xx}^{\mathrm{e}}$ siècles ", Cahier québécois de démographie, 20,1 (1991) : 1-36.

22. J. Mathieu et al., "Peuplement colonisateur au XviII ${ }^{\mathrm{e}}$ siècle dans le gouvernement de Québec", dans R. L. Emerson, W. Kinsley et W. Moser, dir., L'Homme et la nature. Actes de la société canadienne du XVIII siècle (Montréal, 1984), 127-138; J. Mathieu, "Mobilité et sédentarité : stratégies familiales en Nouvelle-France», Recherches sociographiques, 28,2-3 (1987) : 211-227; Y. Beauregard et al., «Famille, parenté et colonisation en Nouvelle-France», Revue d'histoire de l'Amérique française, 39,3 (hiver 1986) : 391-405 ; D. Gauvreau et M. Bourque, "Mouvements migratoires et familles : le peuplement du Saguenay avant 1911 ", Revue d'histoire de l'Amérique française, 42,2 (automne 1988) : 167-192.

23. S. Olson, "Pour se créer un avenir". Stratégies de couples montréalais au XIx ${ }^{\mathrm{e}}$ siècle", Revue d'histoire de l'Amérique française, 51,3 (hiver 1998) : 357-390.

24. R. Brisson, La charpenterie navale à Québec sous le Régime français (Québec, Institut québécois de recherche sur la culture, coll. "Edmond-de-Nevers», $\left.n^{\circ} 2,1983\right)$; J. Burgess, Work, Family and Communauty: Montreal Leather Craftsmen, 1790-1831, thèse de doctorat (histoire), Université du Québec à Montréal, 1987.

25. P. Bischoff, «Des forges du Saint-Maurice aux fonderies de Montréal : mobilité géographique, solidarité communautaire et action syndicale des mouleurs, 1829-1881 ", Revue d'histoire de l'Amérique française, 43,1 (été 1989) : 3-30.

26. C. Dessureault et R. Legault, «Évolution organisationnelle et sociale de la milice sédentaire canadienne : le cas du bataillon de Saint-Hyacinthe, 1808-1830", Journal of the CHA/Revue de la SHC, 8 (1997) : 87-111; C. Dessureault et C. Hudon, op. cit. 
D’autres travaux abordent plus spécifiquement les questions de la cohabitation et de la structure des ménages que nous traiterons, de manière succincte, dans une section ultérieure de cet article. Jusqu’à récemment, la plupart des travaux sur le milieu urbain montréalais ont observé, à partir des recensements nominatifs, un fort pourcentage de cohabitation dans la classe ouvrière montréalaise durant les premières décennies de l'industrialisation ${ }^{27}$. De plus, cette cohabitation dans un même logement qui concernait une assez forte proportion des familles et de personnes non apparentées devenait souvent un indice des conditions de vie difficiles de la classe ouvrière. Dans un article publié dans cette revue en 1992, Gilles Lauzon propose une révision radicale de ce constat grâce à un examen fouillé du travail des recenseurs, à l'emploi combiné de plusieurs sources complémentaires et, surtout, à une nouvelle approche fondée sur le recoupement de données cartographiques, historiques et architecturales du mi$\operatorname{lieu}^{28}$. Cette recherche lui permet de conclure «à coup sûr que les familles de Saint-Augustin, et sans doute aussi celles de Montréal, partagent très rarement des logements entre noyaux familiaux distincts» et, que le cas échéant, cette cohabitation s'effectue presque toujours au sein de la parenté, et de manière souvent temporaire dans le cycle de vie ${ }^{29}$.

Peu après, dans Working Families ${ }^{30}$, Bettina Bradbury adopte cette nouvelle perspective et, surtout, elle propose une interprétation plus générale de la cohabitation et de la structure des ménages en milieu ouvrier durant la période de transition vers le capitalisme. Elle insiste d'abord sur l'évolution constante des structures de ménage et des modèles de cohabitation dans le cycle de vie des familles selon leur taille, l'âge des enfants, les fluctuations de l'économie, les besoins de la famille et de la parenté, de même que selon les variations du statut socioprofessionnel, du statut civil et du genre du chef de ménage. De plus, Bradbury constate le renforcement de la famille nucléaire dans la classe ouvrière montréalaise pendant

27. Notons parmi ces travaux : G. Darroch et M. Ornstein, «Family Co-Residence in Canada in 1871 : Family Life Cycles, Occupations and Networks of Mutual Aid», Historical Papers / Communications historiques (1983) : 30-56; "Family and Household in Nineteenth Century Canada : Regional Patterns and Regional Economies ", Journal of Family History, 9,2 (été 1984) : 158-177.

28. G. Lauzon, "Cohabitation et déménagements en milieu ouvrier montréalais. Essai de réinterprétation à partir du cas du village Saint-Augustin (1871-1881)", Revue d'histoire de l'Amérique française, 46,1 (été 1992) : 115-142.

29. Ibid., 141-142.

30. B. Bradbury, Working Families: Age, Gender and Daily Survival in Industrializing Montreal (Toronto, McClelland \& Stewart Inc., 1993). 
la seconde moitié du $\mathrm{XIX}^{\mathrm{e}}$ siècle. Cependant, la parenté demeure un élément important du tissu social, car elle constitue la principale source de sécurité sociale et d'assistance dans les périodes économiques et personnelles difficiles. La cohabitation même temporaire et la corésidence dans un même immeuble ou dans une même rue sont souvent l'expression de cette entraide familiale et elles révèlent une proximité de la parenté audelà de la famille nucléaire. Enfin, l'auteure souligne que la cohabitation entre parents est plus répandue chez les Canadiens français que parmi les autres groupes ethniques dans le milieu ouvrier montréalais.

Dans un ouvrage récent sur la ville de Saint-Hyacinthe ${ }^{31}$, Peter Gossage examine dans une perspective similaire l'évolution de la structure de ménages et de la proximité résidentielle dans une petite ville ouvrière de la région de Montréal entre 1861 et 1891 . Comme Bradbury, Gossage constate le renforcement de la famille conjugale dans la seconde moitié du $\mathrm{XIX}^{\mathrm{e}}$ siècle et, en suivant une cohorte spécifique de familles durant l'ensemble de la période, il scrute de manière détaillée les interactions entre le cycle de vie des familles et la structure des ménages. Dans cette petite ville ouvrière et encore un peu campagnarde, les ménages complexes ou semi-complexes sont davantage présents chez les bourgeois et les travailleurs non manuels $(50 \%)$, tandis la famille nucléaire est prédominante chez les cultivateurs ( $75 \%$ ), puis chez les journaliers et les travailleurs manuels (60\%).

Cette question de la structure des ménages est également présente dans les recherches en histoire rurale. Dans son ouvrage sur le canton de peuplement récent de Winslow ${ }^{32}$, John I. Little distingue une évolution différentielle de la structure des ménages des Canadiens français et des Écossais de ce canton de 1851 à 1881 . La famille nucléaire progresse davantage chez les premiers, d'environ la moitié à près de $80 \%$ des ménages durant cette période, tandis que le ménage complexe ou multifamilial résiste mieux chez les seconds. Pour Little, la différence entre ces deux groupes, quoique relativement minime, est d'abord liée à leurs comportements migratoires spécifiques plutôt qu'à des modes distincts de transmission des avoirs familiaux qui, dans les deux cas, misent davantage sur le transfert intégral des terres à un héritier, plutôt que sur la division

31. P. Gossage, Families in Transition. Industry and Population in Nineteenth-Century SaintHyacinthe (Montréal, McGill-Queen’s University Press, 1999).

32. J. I. Little, Crofters and Habitants. Settler Society, Economy and Culture in a Quebec Township, 1848-1881 (Montréal, McGill-Queen’s University Press, 1991). 
des patrimoines entre les divers héritiers. Dans une étude sur le mode de succession et le cycle familial dans le comté de Verchères ${ }^{33}$, Diane Gervais identifie la structure des ménages dans cinq paroisses du comté presque essentiellement francophone de Verchères au tournant $\mathrm{du} \mathrm{xx}^{\mathrm{e}}$ siècle. La famille nucléaire y prédomine aussi, soit environ $70 \%$ des ménages, mais à un niveau plus proche des colons écossais que des colons canadiensfrançais de Winslow. Compte tenu des variations importantes de la structure des ménages durant le cycle de vie des familles et de la structure démographique souvent différenciée des populations, D. Gervais propose par ailleurs l'emploi de la méthode de Lutz K. Berkner ${ }^{34}$, qui consiste à isoler la structure spécifique des jeunes ménages, pour mieux évaluer la place réelle des ménages complexes et multifamiliaux.

Toutefois, Gérard Bouchard et ses collaborateurs de l'IREP demeurent assurément la principale source des recherches historiques concernant l'étude de la structure des ménages et des rapports de parenté dans la société rurale du Québec préindustriel ${ }^{35}$. Les travaux de Bouchard sur la société rurale saguenayenne, pour laquelle il dispose d'une banque de données démographiques et généalogiques d'une richesse exceptionnelle, s'insèrent dans une perspective plus globale visant à définir les modalités de fonctionnement et de reproduction du système social dans un terroir de peuplement récent. Selon G. Bouchard, la société rurale saguenayenne se caractérise par une forte dynamique communautaire. Le réseau de sociabilité y est d'abord centré sur la famille, le voisinage, la parenté et la paroisse. D'une part, les rapports de parenté y représentent l'une des composantes déterminantes des rapports sociaux et, surtout, une dimension fondamentale de l'identité paysanne. D'autre part, l'individualisme familial qui s'exprime dans la résistance à la culture et aux institutions extérieures, ainsi que dans la prédominance des ménages nucléaires et dans le modèle dominant de l'exploitant propriétaire, constitue l'autre volet

33. D. Gervais, «Succession et cycle familial dans le comté de Verchères, 1870-1950", Revue d'histoire de l'Amérique française, 50,1 (été 1996) : 69-94.

34. La méthode consiste à isoler la structure des ménages pour les ménages dont le chef de famille est âgé de moins de 35 ans et, dans le cas des ménages complexes ou multifamiliaux, les ménages où l'un des chefs de famille a moins de 35 ans. L. K. Berkner, «The Stem Family and the Developmental Cycle of the Peasant Household: An Eighteen-Century Austrian Exemple», The American Historical Review, 77,2 (avril 1972) : 398-418.

35. La liste des travaux de G. Bouchard et des autres chercheurs de l'IREP qui abordent directement ou indirectement l'étude de la parenté est trop longue pour en dresser de manière exhaustive toutes les références. Voir l'ouvrage synthèse de G. Bouchard, Quelques arpents d'Amérique. Population, économie, famille au Saguenay 1838-1871 (Montréal, Boréal, 1996). 
indissociable de cette identité paysanne qui intègre à la fois, de manière plus ou moins harmonieuse, des valeurs individuelles et collectives.

Par ailleurs, le fonctionnement du système de reproduction sociale, tant les modalités de transmission des terres que les stratégies de migration et de réallocation des ressources d'une génération à l'autre, favorise la formation de réseaux de parenté davantage fondés sur des liens entre les hommes d'une même lignée. La prédominance de la virilocalité et des liens patrilinéaires découle ainsi, dans une perspective comparable à celle de Beaucage, du mode d'organisation économique. Dans cette société rurale, la place des femmes dans les réseaux de solidarité familiale n'est pas du tout comparable à la place observée par Garigue pour le milieu urbain montréalais du milieu du siècle, ni à la place constatée par Roberge dans une communauté semi-rurale des environs de Québec dans les années 1980. De manière générale, dans l'ancienne société rurale saguenayenne, les femmes étaient souvent coupées physiquement de leur famille d'origine et insérées comme étrangères dans une autre famille ou dans un autre réseau familial. Par ailleurs, les conjointes appelées à cohabiter avec les beaux-parents étaient celles qui devaient vivre de manière plus exacerbée les tensions quotidiennes liées à cette émigration familiale ${ }^{36}$.

De cette littérature scientifique, nous dégageons certains constats généraux auxquels nous pourrons apporter de nouveaux éléments de réflexion et de nouveaux critères de vérification empirique. Ainsi, les auteurs s'entendent sur l'importance des rapports de parenté dans la société québécoise préindustrielle et sur la forte densité des réseaux de parenté, plus spécifiquement dans le monde rural. Plusieurs auteurs conviennent d'une différenciation selon le genre portant tantôt sur la notion subjective du rapport à la parenté, tantôt sur celle plus objective de la proximité résidentielle des réseaux de parenté. Selon certains, cette différenciation des réseaux de parenté renvoie plus spécifiquement au mode d'organisation économique, soit au rapport à la propriété des biens de production, soit au mode de transmission des biens. Plus récemment, on évoque aussi une différenciation des réseaux selon les statuts sociaux. Par ailleurs, ces divers travaux identifient une variété complexe et différenciée de structures de ménage selon les milieux étudiés, mais les auteurs s'entendent assez généralement sur la prédominance de la famille nucléaire. Ceux-ci proposent tour à tour des modèles d'interprétation spécifiques liés au cycle de vie, aux changements économiques, aux comportements migratoires ou aux modes 
de transmission des terres pour mieux saisir la nature et l'évolution de ces structures de ménage dans leur milieu spécifique. Notre enquête intègre donc sous forme de questions ouvertes ces divers constats et ces diverses interrogations de la littérature scientifique. Enfin, nous empruntons à certains de ces auteurs des notions dont la définition prend vraiment un sens dans ce champ spécifique de la recherche comme la division entre la parenté proche et la parenté éloignée, ou encore la distinction entre la cohabitation et la corésidence.

\section{LE TERRAIN D'ENQUÊTE}

La paroisse de Saint-Antoine-de-Lavaltrie est située le long du fleuve SaintLaurent, sur la rive nord, à environ $50 \mathrm{~km}$ au nord-est de Montréal (voir carte). En 1861, cette paroisse compte 1308 habitants répartis dans 203 maisonnées. Ce secteur fait partie des plus anciens terroirs de peuplement de la plaine de Montréal. La concession des premières terres remonte à la

\section{CARTE 1}

\section{La paroisse de Saint-Antoine de Lavaltrie}

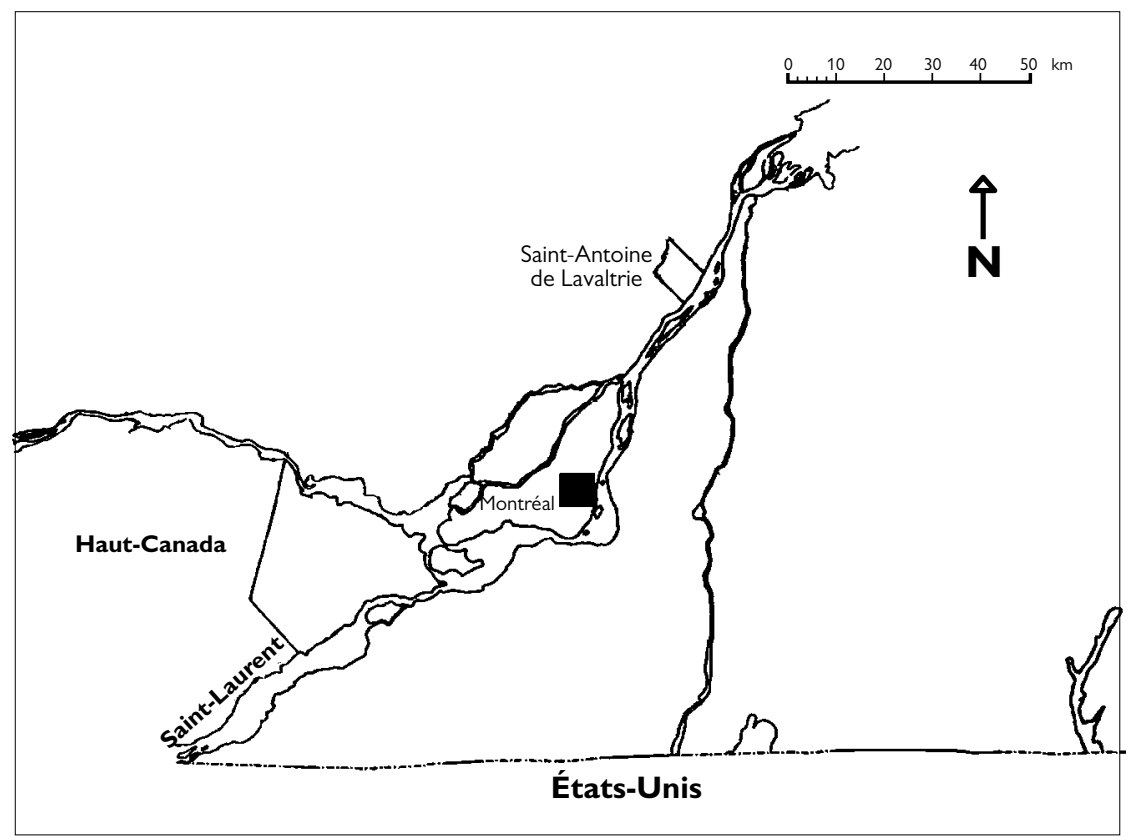

Figure produite à partir de : $\mathrm{S}$. Courville dir., Paroisses et municipalités de la région de Montréal au XIX ${ }^{\mathrm{e}}$ siècle (|825-186I) (Québec, PUL, 1988), Figure 13. 
fin du XVII ${ }^{\mathrm{e}}$ siècle, peu après la concession d'une seigneurie au sieur de Lavaltrie en $1672^{37}$. La paroisse de Saint-Antoine-de-Lavaltrie est officiellement créée en 1722.

$\mathrm{Au} \mathrm{xvIII}{ }^{\mathrm{e}}$ siècle, le peuplement du secteur progresse lentement à partir du noyau initial de colons, établis sur les bords du fleuve, vers les côtes de l'intérieur et, en 1791, la population de la paroisse atteint 500 habitants. Dans les premières décennies du $\mathrm{xIx}^{\mathrm{e}}$ siècle, Saint-Antoine-de-Lavaltrie connaît, à l'instar de nombreux autres secteurs de la vallée du SaintLaurent, une saturation progressive de l'espace agraire. En 1831, la paroisse compte environ 1000 habitants. Elle représente désormais un terroir entièrement concédé et occupé, c'est-à-dire un terroir plein qui atteindra bientôt un plateau démographique. Par ailleurs, à partir du début du $\mathrm{xIx}^{\mathrm{e}}$ siècle, la maturation du terroir et l'essor de l'économie de marché favorisent la différenciation sociale au sein des classes populaires rurales, soit l'accroissement des inégalités dans la société paysanne, l'essor des artisans et l'émergence d'un prolétariat rural. Cette diversification des statuts sociaux s'accentue alors que l'agriculture demeure la principale activité économique dans la communauté rurale. En 1861, l'agriculture reste l'activité dominante à Saint-Antoine-de-Lavaltrie. Les principales productions agricoles sont l'avoine, le seigle, l'orge et les pommes de terre. Le blé froment y représente alors une culture secondaire. Par ailleurs, les exploitations produisent une gamme variée d'autres produits complémentaires tels le beurre, les textiles domestiques et le sucre d'érable. Les cultivateurs de Lavaltrie possèdent en moyenne un cheptel assez imposant : 14 bovins dont 5 bœufs et 4 vaches laitières, 3 chevaux, 8 moutons et 3 porcs. La superficie moyenne des propriétés rurales, de 121,4 arpents, dont près de 75 arpents sont défrichés, de même que la valeur moyenne des terres, soit 2937 \$, témoignent de l'aisance relative de cette paroisse rurale de la vallée du Saint-Laurent ${ }^{38}$.

37. Brevet de concession du fief et seigneurie de Lavaltrie, dans P.-G. Roy, Inventaire des concessions en fief et seigneurie, fois et hommages, et aveux et dénombrements (Québec/Beauceville, Archives du Québec/L’éclaireur Limitée, 1927), II : 243-244.

38. Pour comparaison, en 1861, la valeur moyenne des terres est inférieure à $600 \$$ dans le canton de Winslow, une région de peuplement récent des Cantons de l'Est (501 \$ pour les cultivateurs canadiens-français et 568 \$ pour les cultivateurs écossais). Voir J. I. Little, op. cit., 147. 


\section{LES SOURCES ET LA MÉTHODOLOGIE}

Nous avons utilisé deux sources principales : le recensement nominatif de 1861 et les divers répertoires de mariage disponibles ${ }^{39}$. Le recensement nominatif a d'abord permis d'identifier les maisonnées, la structure des ménages et les chefs de ménage de la paroisse de Lavaltrie en 1861. Les mentions de profession et de statut ont ensuite été utiles pour classer les chefs de ménage dans diverses catégories socioprofessionnelles. Nous avons également jumelé les données du recensement agraire à celles du recensement nominatif et nous avons retenu plus spécifiquement la valeur des propriétés foncières pour déterminer quatre groupes de cultivateurs parmi l'exemple des exploitants propriétaires de cette paroisse.

Ces catégories de cultivateurs ont été plus précisément déterminées en calculant l'écart type de la valeur des terres possédées par les cultivateurs de cette paroisse : $2476 \$$. Nous avons ensuite identifié chacune de ces quatre catégories de cultivateurs selon la valeur de leurs terres :

Catégorie 1. Moins de 1238 (moins de la moitié de l'écart type)

Catégorie 2. De 1238 à 2476 (de la moitié à la totalité de l'écart type)

Catégorie 3. De 2476 à $4952 \$$ (de la totalité de l'écart type à son double)

Catégorie 4. Plus de 4952\$(plus du double de l'écart type)

Cette classification est essentiellement opératoire. Nous ne prétendons pas définir de cette manière des classes sociales distinctes parmi les cultivateurs. Grâce à l'utilisation de ces catégories, nous pouvons toutefois mieux tenir compte des inégalités socio-économiques au sein de la paysannerie dans l'analyse des données sur les réseaux de parenté, d'autant plus que les clivages dans la valeur des propriétés paraissent suffisamment significatifs.

Nous avons ensuite déterminé, à partir des dossiers généalogiques et de certaines données tirées du recensement nominatif, l'étendue, la nature et les limites sociales de ces liens de parenté selon le statut socioprofessionnel des chefs de ménage en 1861 . Nous voulons ainsi vérifier la distance ou la proximité des liens familiaux à l'intérieur et entre les groupes socioprofessionnels, de même que les liens possibles entre les statuts sociaux et l'étendue des réseaux de parenté dans cette paroisse rurale. Par ailleurs, la

39. Nous avons d'abord utilisé le fichier Loiselle et le répertoire des mariages Drouin dans lesquels nous pouvons retracer une bonne partie des mariages célébrés dans toutes les paroisses catholiques du Québec des origines à la fin du Xix ${ }^{\mathrm{e}}$ siècle. Nous avons ensuite eu recours aux répertoires de mariage des comtés de Berthier, de Joliette et de l'Assomption, de même qu'aux répertoires de diverses paroisses environnantes de la région de Montréal. 
différenciation des réseaux de parenté à l'intérieur du groupe paysan, selon la valeur des patrimoines fonciers, a fait l'objet d'une attention particulière.

Nous avons constitué les dossiers généalogiques de chacun des ménages en retraçant dans les divers répertoires de mariage disponibles (le fichier Loiselle, le répertoire Drouin et les divers répertoires de mariage régionaux et paroissiaux) l'union et, le cas échéant, les unions antérieures du chef de ménage et de sa conjointe, les unions de leurs parents et de tous leurs grands-parents respectifs. Nous avons également identifié les parents et les grands-parents du curé de la paroisse puisque nous le considérons dans cette enquête comme un chef de ménage. Par contre, nous n'avons pu retracer les ascendants et les liens de parenté de sept ménages de la paroisse, incluant les parents de deux institutrices qui vivaient seules. Nous avons ainsi réussi à établir des fiches de généalogie ou de parenté pour 196 des 203 ménages de la paroisse dans lesquelles nous avons indiqué les mariages des conjoints du couple principal d'un ménage, de leurs parents et de leurs grands-parents ${ }^{40}$. Nous avons ainsi réuni une masse considérable de données nous permettant, grâce à un logiciel de généalogie relativement simple ${ }^{41}$, d'identifier presque tous les liens de parenté, jusqu'au cousinage en ligne directe ou par alliance, entre la plupart des chefs de ménage de Lavaltrie en 1861.

Nous avions également retracé et traité les mêmes données généalogiques concernant les deux conjoints de couples vivant en cohabitation avec le couple principal d'un ménage ${ }^{42}$, de même que les liens familiaux concernant les veuves et les veufs vivant en corésidence. Cependant, nous avons ensuite exclu ces fiches des calculs sur les liens de parenté. Nous avons préféré limiter notre enquête généalogique aux chefs de ménage et à leurs conjointes compte tenu de la difficulté d'identifier avec certitude tous les liens de parenté, jusqu'au cousinage, de même que l'état civil de toutes

40. Nous avons compilé 186 fiches complètes et 10 fiches partielles concernant des couples dont l'un des parents ou l'un des grands-parents n'ont pas été identifiés.

41. Nous avons utilisé pour cette recherche le logiciel «Reunion» de la société Leister Production (version Macintosh 6.0). Ce logiciel de généalogie permet d'identifier dans un fichier associant les 196 fiches de généalogie familiale de Saint-Antoine-de-Lavaltrie toutes les personnes apparentées à un individu spécifique du même ficher en spécifiant le ou les liens de parenté.

42. Il est quelquefois difficile de déterminer précisément le chef d'un ménage. À Lavaltrie, nous avons ainsi rencontré quatre maisonnées impliquant la cohabitation de deux unités conjugales, non apparentées l'une à l'autre. Dans chacune de ces maisonnées, deux hommes mariés déclaraient une profession. Nous avons alors choisi comme le chef de chacun de ces ménages le premier homme marié mentionné par le recenseur. Dans ces quatre cas, il s'agissait aussi du plus âgé de deux hommes mariés du ménage. 
les personnes adultes vivant en cohabitation dans la paroisse de Lavaltrie, en 1861. Nous utiliserons toutefois certaines données complémentaires pour traiter, entre autres, des parents proches vivant en cohabitation familiale.

\section{LA STRUCTURE DES MÉNAGES ET LA COHABITATION FAMILIALE}

Notre étude porte sur les liens de parenté entre l'ensemble des chefs de ménage d'une paroisse rurale. Toutefois, nous devons d'abord vérifier la structure des ménages dans la paroisse étudiée en identifiant la nature des liens de parenté à l'intérieur même des ménages. Les premiers liens de parenté, les plus proches et les plus influents dans la vie quotidienne d'une personne, sont certainement ceux de son environnement immédiat.

Nous avons utilisé la typologie des ménages de l'IREP ${ }^{43}$, elle-même construite à partir des travaux de Peter Laslett ${ }^{44}$. Cette typologie comprend quatre types :

1. les ménages sans noyau conjugal : ces ménages sont constitués d’individus apparentés ou non, célibataires ou veufs, sans liens conjugaux les uns aux autres et sans liens de filiation verticale directe (père/enfants ou mère/enfants);

2. les ménages simples ou nucléaires: ces ménages sont formés d'un noyau familial unique constitué soit d'un couple marié sans enfant(s), soit d'un couple marié avec enfant(s), soit d'un veuf ou d'une veuve avec enfant(s);

3. les ménages élargis ou semi-complexes: ces ménages sont formés, comme les ménages simples, d'un noyau familial unique et d'une ou de plusieurs autres personnes apparentées ou non partageant la même résidence;

4. les ménages multifamiliaux ou complexes : ces ménages sont formés de deux ou de plusieurs noyaux familiaux apparentés ou non les uns aux autres.

Dans cette section, nous tenterons donc de présenter les spécificités de la structure des ménages de la paroisse de Lavaltrie en confrontant nos

43. G. Bouchard, et J. Larouche, Enquête sur la structure des ménages saguenayens. Présentation de la typologie (Document de l'IREP, $\mathrm{n}^{\circ}$ II, C-187, juin 1991), $11 \mathrm{p}$.

44. P. Laslett, «La famille et le ménage : approches historiques», Annales ESC, 27,4-5 (juilletoctobre 1972) : 847-872. 
données avec les résultats de travaux antérieurs sur la société rurale québécoise et, plus spécifiquement, ceux de G. Bouchard et de D. Gervais qui ont utilisé la même typologie. Nous référerons aussi à d'autres recherches en prenant soin d'employer exclusivement des données comparables.

En 1861, la paroisse de Lavaltrie compte six ménages sans noyau conjugal regroupant la maison du curé, celle d'une institutrice et celles de veufs ou de veuves demeurant tantôt seules, tantôt avec des personnes non apparentées (tableau 1). La majorité des ménages de cette paroisse sont de type simple ou nucléaire. Néanmoins, à $57 \%$, le pourcentage des ménages simples ou nucléaires est beaucoup moins élevé dans cette vieille paroisse de la plaine de Montréal que dans la région de peuplement récent du Saguenay Lac Saint-Jean où, à la fin du $\mathrm{xIx}^{\mathrm{e}}$ et au début du $\mathrm{xx}^{\mathrm{e}}$ siècle, il représente près de $80 \%$ de l'ensemble des ménages ${ }^{45}$. Il est également moins élevé que dans la plupart des autres milieux ruraux où cette question a jusqu'à présent été étudiée, à l'exception des colons canadiensfrançais du canton de Winslow en $1852^{46}$. Par ailleurs, la structure des

TABLEA U 1

Structure comparée des ménages à Lavaltrie en 1861, à Verchères en 1891 et au Saguenay de 1881 à 1912

\begin{tabular}{l|c|c|c|c|c|c}
\hline & \multicolumn{2}{|c|}{$\begin{array}{c}\text { Lavaltrie } \\
\mathbf{1 8 6 1}\end{array}$} & \multicolumn{2}{c|}{$\begin{array}{c}\text { Verchères } \\
\mathbf{1 8 9 1}\end{array}$} & \multicolumn{2}{c}{$\begin{array}{c}\text { Saguenay } \\
\mathbf{1 8 8 1 - 1 9 1 2}\end{array}$} \\
\hline Types de ménages & Nombre & $\%$ & Nombre & $\%$ & Nombre & $\%$ \\
\hline $\begin{array}{l}\text { 1. Ménages sans noyau } \\
\text { conjugal }\end{array}$ & 6 & 3,0 & 67 & 5,9 & 40 & 1,2 \\
$\begin{array}{l}\text { 2. Ménages simples } \\
\begin{array}{l}\text { 3. Ménages } \\
\text { semi-complexes }\end{array}\end{array}$ & 116 & 57,1 & 829 & 74,1 & 2546 & $\begin{array}{r}78,7 \\
\text { M. Ménages complexe }\end{array}$ \\
$\begin{array}{l}\text { 4. } \\
\text { Total }\end{array}$ & 20 & 90,0 & 115 & 10,3 & 318 & 9,8 \\
\hline
\end{tabular}

Source : Recensement nominatif de I86I; G. Bouchard, Quelques arpents d'Amérique. Population, économie, famille au Saguenay 1838-197I (Montréal, Boréal, 1996), 265; D. Gervais, «Succession et cycle familial dans le comté de Verchères, I870-1950», Revue d'histoire de l'Amérique française, 50,I (été 1996): 78.

45. G. Bouchard, op. cit., 265.

46. En 1852, les familles nucléaires représentent environ 59\% des ménages chez les Canadiens français de Winslow. Toutefois, ce pourcentage s'accroît rapidement dans les décennies suivantes. En 1881, le pourcentage de ce type de ménage dans ce même groupe atteint $80 \%$. J. I. Little, op. cit., 107. 
ménages dans le comté voisin de Verchères, au tournant du $\mathrm{xx}^{\mathrm{e}}$ siècle, se situe à un niveau plus proche de la région du Saguenay que celui de la paroisse de Lavaltrie ${ }^{47}$.

L'ancienneté du terroir influe sans doute sur la forte proportion de ménages complexes et semi-complexes dans la paroisse de Lavaltrie. Dans cette vieille paroisse de la vallée du Saint-Laurent, l'unité de résidence ne correspond pas autant au noyau conjugal qu'au Saguenay, une région de colonisation récente. Cependant, ce facteur ne semble pas si déterminant lorsqu'on observe les données du comté voisin de Verchères.

La principale spécificité de la structure des ménages de Lavaltrie est plus précisément l'importance des ménages semi-complexes qui, à l'instar des ménages simples, sont constitués d'un seul noyau conjugal. Ce noyau conjugal est enrichi tantôt de la présence de parents, tantôt de la présence de personnes étrangères à la famille, souvent des employés ou des serviteurs. Dans le cas de la cohabitation de l'un des membres de la parenté proche, surtout l'un des vieux parents, ce type de ménage constitue souvent la forme dérivée d'un ménage complexe ou multifamilial. Dans l'autre cas, la rationalité de ce type de ménage semble beaucoup plus proche de celle du ménage nucléaire. Nous devrons donc examiner attentivement la ou les rationalités sous-jacentes à la présence de ces ménages semi-complexes.

Dans son ouvrage sur le canton de Winslow, J. I. Little souligne l'absence d'impact d'un mode préférentiel de transmission des terres sur la structure des ménages des cultivateurs canadiens-français de cette région de peuplement récent ${ }^{48}$. Dans un terroir de peuplement plus ancien, à Lavaltrie, nous relevons toutefois plusieurs indices d'une relation entre le poids relativement important des ménages complexes et semi-complexes dans la structure des ménages et un mode de transmission des patrimoines qui est principalement axé sur la donation préférentielle de la terre familiale à l'un des fils ${ }^{49}$.

Cette paroisse compte une vingtaine de ménages complexes ou multifamiliaux sur 203 ménages. Or, 15 de ces 20 ménages complexes impliquent la cohabitation de familles paysannes unies par des liens patrilinéaires :

47. D. Gervais, op. cit., 78.

48. J. I. Little, op. cit.

49. La donation de la terre familiale à l'un des fils a longtemps été associée à la saturation du terroir. Cependant, S. Dépatie a bien montré que l'intensification de ce mode de transmission des terres n’est pas nécessairement liée au terroir plein. S. Dépatie, «La transmission du patrimoine dans les terroirs en expansion : un exemple canadien au xvIII ${ }^{\mathrm{e}}$ siècle", Revue d'histoire de l'Amérique française, 44,2 (automne 1990) : 171-198. 
tantôt des parents âgés, à la retraite, cohabitant avec la famille de leur fils; tantôt un jeune couple, avec ou sans enfant, vivant dans la demeure des parents de l'époux. Ce mode de cohabitation du père et du fils ou de la bellemère et de la bru renvoie directement à un système de reproduction sociale selon lequel les parents privilégient l'établissement de l'un de leurs garçons sur la terre familiale, souvent nommée le vieux bien $^{50}$. Un autre ménage de type complexe ou multifamilial concerne plus spécifiquement un mode de cohabitation similaire, au sein du monde artisanal, entre la famille du menuisier Elpide Brault et ses deux parents. Toutefois, ce mode de cohabitation ne se vérifie pas parmi les autres ménages complexes de la paroisse n'appartenant pas à la paysannerie. L'un de ces autres ménages complexes réunit deux sœurs et leurs époux, tous deux journaliers, tandis que les quatre autres impliquent la cohabitation de familles n'étant pas directement apparentées.

À Lavaltrie, les ménages semi-complexes sont très fréquents puisque la paroisse en compte 61 sur 203 ménages. Nous devons toutefois scinder ce type de ménages en deux catégories distinctes selon les liens de parenté des personnes additionnelles avec les membres du noyau principal. Nous pouvons ainsi regrouper dans la première catégorie (le type 3a), les ménages dans lesquels au moins une des personnes additionnelles est directement apparentée ou alliée à l'un des conjoints du noyau principal. Par contre, nous identifions comme deuxième catégorie (le type $3 \mathrm{~b}$ ) les ménages où toutes les personnes additionnelles n'ont aucun lien de parenté direct ou indirect, facilement repérable, avec l'un ou l'autre des deux conjoints du noyau principal.

Chez les cultivateurs, le mode de cohabitation de type 3 a demeure, à l'instar du type de ménage complexe ou multifamilial, fortement tributaire du système de reproduction sociale puisque la personne additionnelle, présente dans le noyau conjugal, y est le plus souvent le père ou la mère du chef de ménage. Après le décès de l'un des deux parents, le survivant continue de résider chez le garçon donataire. Le type de ménage se modifie, du type 4 au type 3a, mais la rationalité du mode de cohabitation

50. Le vieux bien ne constitue pas nécessairement la seule composante du patrimoine foncier d'une famille. Celui-ci peut comprendre une ou plusieurs terres additionnelles tandis que les avoirs familiaux comprennent habituellement d'autres types de biens que des terres. De plus, la transmission des biens d'une famille, d'une génération à l'autre, peut s'effectuer selon plusieurs modalités, à divers moments du cycle de vie. Dans cette perspective, la transmission préférentielle de la terre familiale ou du vieux bien à l'un des fils peut s'avérer, dans de nombreux cas, l'une des facettes d'un système plus complexe d'héritage et de reproduction sociale. 
demeure la même. Ce mode de cohabitation implique 16 ménages paysans, un ménage d'artisan et un ménage de journalier. Par ailleurs, la présence d'un autre parent comme personne additionnelle, dans le noyau principal, est constatée dans trois autres ménages paysans, dans un autre ménage d'artisan et surtout dans six autres ménages de journaliers où prédominent, à l'inverse, des liens matrilinéaires entre la personne additionnelle et le couple principal : la personne additionnelle est alors la mère ou la sœur de l'épouse du chef de ménage. Certes, la cohabitation d'une personne apparentée ne repose pas toujours sur des fondements successoraux même lorsqu'il s'agit de l'un des parents du chef de ménage. Néanmoins, la prédominance de liens de parenté patrilinéaires dans le milieu paysan, par opposition à la variété plus grande des liens de parenté constatés dans les ménages complexes et semi-complexes des autres milieux socioprofessionnels, vient accréditer la relation étroite entre le système de reproduction sociale et le mode de cohabitation familiale parmi les ménages paysans de Lavaltrie.

Dans le milieu paysan, les deux types de ménages semi-complexes et complexes impliquant la cohabitation des vieux parents ou de l'un d'eux avec l'un de leurs fils et sa conjointe sont davantage présents dans les deux catégories supérieures de cultivateurs fixées selon la valeur des patrimoines fonciers (tableau 2). D'une part, la capacité de production d'une exploitation agricole constitue un élément important pour subvenir aux besoins d'un ménage élargi. D'autre part, la valeur d'un patrimoine foncier incite sans doute certains garçons, ainsi que leur conjointe, à assumer plus facilement les contraintes et les charges liées à l'héritage

TABLEAU 2

La cohabitation des vieux parents et de l'un de leurs garçons selon le type de ménages et selon la valeur des terres, à Lavaltrie, en 1861

\begin{tabular}{l|c|c|c|c|c}
\hline & \multicolumn{4}{|c|}{$\begin{array}{c}\text { Catégories de cultivateurs selon la valeur des } \\
\text { terres possédées (en dollars) }\end{array}$} & \\
\hline $\begin{array}{l}\text { Types de ménages } \\
\text { - de 1238 } \$\end{array}$ & $\begin{array}{c}\mathbf{2} \\
1238-2476\end{array}$ & $\begin{array}{c}\mathbf{3} \\
2476-4952\end{array}$ & $\begin{array}{c}\mathbf{4} \\
+\mathrm{de}\end{array}$ 4952 $\$$ & $\begin{array}{c}\text { Nombre } \\
\text { total }\end{array}$ \\
\hline $\begin{array}{l}\text { Ménages } \\
\text { semi-complexes }\end{array}$ & 3 & 2 & 4 & 7 & 16 \\
Ménages complexes & 4 & - & 5 & 6 & 15 \\
\hline Sous-total & 7 & 2 & 9 & 13 & 31 \\
\hline Nombre total & 34 & 30 & 29 & 28 & 121 \\
\hline
\end{tabular}


paternel ${ }^{51}$. Toutefois, nous retrouvons aussi les mêmes modes de cohabitation parmi certains cultivateurs possédant des patrimoines de moindre valeur, des terres peu susceptibles de produire les surplus nécessaires à la subsistance d'un ménage élargi et nettement moins attrayantes comme héritage familial.

Par ailleurs, la majorité des ménages semi-complexes de la paroisse de Lavaltrie comportent la présence d'une ou de plusieurs personnes qui ne sont pas directement apparentées à l'un ou à l'autre des conjoints du noyau principal. L'un des ménages complexes ou multifamiliaux compte aussi une personne additionnelle étrangère aux deux noyaux familiaux du ménage. Dans plusieurs cas, le recenseur note simplement le statut d'étranger ou d'étrangère d'une personne dans le ménage visité. Dans 18 ménages de la paroisse, la ou les personnes additionnelles sont toutefois explicitement identifiées comme serviteurs ou comme servantes au sein de ménages disposant d'un statut social plus élevé ou de meilleurs moyens financiers. Dans deux autres cas, liés au monde artisanal, ces corésidents étrangers à la famille sont des personnes partageant le même statut socioprofessionnel que le chef de ménage, sans doute des apprentis ou des compagnons. Ainsi, comme dans la ville de Saint-Hyacinthe étudiée par P. Gossage, la présence de serviteurs ou de servantes est souvent liée au statut social ou à la fonction économique d'un ménage. La famille du médecin de la paroisse, celle de l'un des trois marchands et celle de l'un des trois bourgeois bénéficient ainsi de la présence d'une servante, tandis que le ménage de l'hôtelier compte comme personnes additionnelles à la famille immédiate un serviteur, une servante et une "étrangère». Mais, dans la paroisse de Lavaltrie, la plupart des serviteurs et des servantes sont recensés dans des ménages paysans, et plus généralement parmi les plus importants propriétaires fonciers du groupe ${ }^{52}$. La prospérité d'une partie assez importante des cultivateurs de Lavaltrie constituerait donc l'autre

51. Pour M. Verdon, à partir du xix ${ }^{\mathrm{e}}$ siècle, le développement des autres secteurs de l'économie et l'émigration aux États-Unis rendent plus difficile le recrutement d'un héritier désireux d'assumer la succession sur la terre familiale. Dans un contexte d'agriculture menacée, la transmission préférentielle de la terre familiale à l'un des fils vise alors à retenir au moins l'un de ceuxci dans l'agriculture. Par ailleurs, ce mode de reproduction familiale suscite des contraintes et des conflits liés à la cohabitation familiale des donateurs et des donataires, et plus spécifiquement entre les belles-mères et les brus. Voir M. Verdon, «Autour de la famille souche. Essai d'anthropologie conjecturale ", Anthropologie et sociétés, 11,1 (1987) : 137-160.

52. Sur 14 ménages paysans comptant une ou des personnes additionnelles explicitement identifiées comme serviteurs ou comme servantes, 11 possèdent des terres d'une valeur supérieure à $4000 \$$. 
facteur explicatif de cette forte proportion de ménages semi-complexes dans cette paroisse.

L'analyse de cette structure des ménages et, surtout, la confrontation de nos données avec celles d'autres recherches révèlent la complexité de cette question et elles démontrent la difficulté d'établir un seul modèle d'interprétation globale, de nature unidimensionnelle, pour l'ensemble des milieux ruraux ou des milieux urbains. Chaque terrain d'enquête a ses spécificités démographiques, économiques et sociales et le chercheur doit absolument tenir compte de cette complexité du réel.

L'ancienneté du terroir, les modes successoraux, les comportements migratoires et le développement des exploitations agricoles sont autant de facteurs qui peuvent influer, dans un sens ou dans l'autre, sur la structure des ménages dans les campagnes. Dans certains contextes, l'historien peut sans doute privilégier l'un de ces facteurs au détriment des autres. Dans d'autres contextes, ces mêmes facteurs sont plutôt liés les uns aux autres. La consolidation des exploitations agricoles peut éventuellement permettre à certains cultivateurs d'employer une main-d'œuvre extérieure à la famille, soit pour les travaux agricoles, soit pour les services domestiques. Cette consolidation rend aussi plus attrayante la transmission des terres par voie de donation, tandis que la densification de la population dans l'ensemble d'une région, en diminuant l'espace nécessaire à l'établissement de tous les enfants d'une famille dans une même paroisse, entraîne le renforcement de cette même transmission préférentielle à l'un des héritiers et l'essor des ménages complexes et semi-complexes dans le milieu paysan. Par ailleurs, ce mode successoral, impliquant l'exclusion des autres héritiers, entraîne l'émigration à l'extérieur de la paroisse d'une partie des autres enfants et la dispersion géographique d'une fraction de la parenté proche. Cependant, l'exclusion successorale n'est pas la seule, ni même la principale cause de l'émigration des ruraux qui, dans la seconde moitié du $\mathrm{XIX}^{\mathrm{e}}$ siècle, a assurément de fortes incidences sur l'évolution des structures sociales, des structures de ménage et, surtout, des réseaux de parenté à l'extérieur et à l'intérieur des paroisses.

\section{LA PARENTÉ ENTRE LES CHEFS DE MÉNAGE DE LAVALTRIE}

La cohabitation familiale à l'intérieur des ménages complexes ou semicomplexes constitue un élément important pour vérifier la proximité de la parenté dans l'espace paroissial. Mais ces données sur la cohabitation familiale ne livrent qu'une partie de l'importance des réseaux de parenté dans la vie quotidienne des ménages. Les divers ménages de Lavaltrie, 
qu'ils soient sans noyau conjugal, nucléaires, semi-complexes ou complexes, font partie d'un réseau de parenté plus étendu ${ }^{53}$. Nous avons voulu déterminer l'importance de ces réseaux, à l'intérieur de la communauté locale, en vérifiant les liens familiaux entre les divers chefs de ménage de Lavaltrie, tant la parenté directe que celle issue des alliances matrimoniales.

Nous pouvons d'abord distinguer la parenté proche, directe ou par alliance, qui comprend les enfants, les petits-enfants, les grands-parents, les parents, les frères et les beaux-frères des chefs de ménage et de leur conjointe. Chaque chef de ménage compte en moyenne 3 liens de parenté proche dans la paroisse. Nous pouvons ensuite considérer une parenté élargie intégrant à la parenté proche les neveux, les oncles et les cousins directs ou par alliances matrimoniales du chef de ménage et de sa conjointe. Chaque chef de ménage de Lavaltrie compte alors 11 liens de parenté parmi l'ensemble des chefs de ménage de la paroisse : c'est-à-dire un peu moins de $6 \%$ d'entre eux. En 1861, la plupart des chefs de ménage de Lavaltrie peuvent donc compter sur la proximité résidentielle de quelques parents proches et d'un nombre un peu plus considérable de parents plus éloignés. Ces données permettent toutefois de mieux définir l'étendue réelle des réseaux de parenté qui, même à un niveau élargi jusqu'aux cousins directs ou par alliance, réunissent au sein d'un même cercle familial une proportion relativement faible des ménages d'une même paroisse.

Par ailleurs, l'étendue de ces réseaux de parenté est très variable d'un chef de ménage à l'autre (tableau 3). Ainsi, nous n'avons retracé aucun lien de parenté proche pour environ $12 \%$ des chefs de ménage de Lavaltrie, et deux liens et moins pour plus de $50 \%$ de ces chefs de ménage. Par contre, environ $20 \%$ de ceux-ci comptent au moins cinq parents proches parmi l'ensemble de tous les chefs de ménage de la paroisse et le plus grand nombre de parents proches identifiés dans seulement deux cas est de 10 liens. Cette diversité des réseaux est également présente lorsque nous considérons un niveau de parenté élargie. Certes, l'absence totale

53. Ainsi, alors que plusieurs recherches venaient de démontrer la prédominance des ménages de type nucléaire dans les sociétés occidentales, M. Segalen prenait soin de souligner que ces unités familiales ne constituaient pas pour autant des entités totalement autonomes et réaffirmait ainsi l'importance de la famille et des réseaux de parenté dans l'organisation sociale. Voir M. Segalen, «Nuclear is not Independent: Organisation of the Household in the Pays Bigouden du Sud in the Nineteenth and Twentieth Century", dans R. M. Netting, R. W. Wilk, E. J. Arnould, dir., Comparative and Historical Studies of the Domestic Group (Berkeley, University of California Press, 1984), 163-186. 
TABLEA U 3

La répartition des chefs de ménage selon le nombre de liens de parenté parmi l'ensemble des chefs de ménage de Lavaltrie, 1861

\begin{tabular}{c|c|c|c|c|c}
\hline $\begin{array}{c}\text { Nombre des liens } \\
\text { de parenté }\end{array}$ & \multicolumn{2}{|c|}{$\begin{array}{c}\text { PARENTÉ } \\
\text { PROCHE }\end{array}$} & $\begin{array}{c}\text { Nombre des liens } \\
\text { de parenté }\end{array}$ & \multicolumn{2}{c}{$\begin{array}{c}\text { PARENTÉ } \\
\text { ÉLARGIE }\end{array}$} \\
\hline & $\begin{array}{c}\text { Nombre } \\
\text { de cas }\end{array}$ & $\%$ & & $\begin{array}{c}\text { Nombre } \\
\text { de cas }\end{array}$ & $\%$ \\
\hline \multirow{2}{*}{ aucun } & 24 & 12,2 & aucun & 7 & 3,6 \\
1 & 36 & 18,4 & 1 à 5 & 59 & 30,1 \\
2 & 41 & 20,9 & 6 à 10 & 41 & 20,9 \\
3 & 27 & 13,8 & 11 à 15 & 30 & 15,3 \\
4 & 27 & 13,8 & 16 à 20 & 22 & 11,2 \\
5 et + & 41 & 20,9 & 21 et + & 37 & 18,9 \\
\hline
\end{tabular}

- Parenté proche : parenté directe ou par alliances matrimoniales, petits-enfants, enfants, parents, grands-parents, frères, beaux-frères du chef de ménage et de sa conjointe.

- Parenté élargie : la parenté proche, plus les neveux, les oncles et les cousins directs ou par alliances matrimoniales du chef de ménage et de sa conjointe.

de liens de parenté jusqu'aux cousins directs ou par alliance dans la communauté locale demeure marginale. Néanmoins, environ 30\% des ménages enregistrent 5 liens et moins de parenté élargie et plus de $50 \%$ des ménages, 10 liens et moins. Par contre, près de $20 \%$ des ménages comptent plus de 20 liens de parenté élargie et le plus grand nombre de ce type de liens de parenté retracés pour un même ménage, à Lavaltrie, est de 41.

\section{LA PARENTÉ SELON LE GENRE}

La cohabitation familiale dans les ménages complexes et semi-complexes privilégie la proximité et la solidarité masculine au sein de la famille. Dans les terroirs relativement récents du Saguenay Lac-Saint-Jean où la cohabitation n'a pas la même ampleur qu'à Lavaltrie, G. Bouchard note des écarts importants de proximité résidentielle selon le genre des enfants. "Davantage que les garçons, les filles étaient susceptibles de s'éloigner de leur famille d'origine pour être intégrées à la dynamique de leur bellefamille ${ }^{54}$." Le taux de corésidence dans la même paroisse des membres 
d'une famille était ainsi beaucoup plus élevé chez les garçons que chez les filles.

À Lavaltrie, en 1861, dans un terroir de peuplement plus ancien, la proximité différentielle des enfants selon le genre intervient davantage pour la cohabitation familiale que pour la corésidence du groupe familial dans la même paroisse. Certes, de manière générale, les liens de parenté y demeurent légèrement plus nombreux du côté de la famille de l'époux (5,95 liens) que du côté de la famille de l'épouse (5,42 liens). Par contre, parmi les descendants des deux conjoints, les gendres sont légèrement plus nombreux parmi l'ensemble des chefs de ménage de la paroisse que les fils (le ratio est de 0,17 pour les gendres et de 0,16 pour les fils). Toutefois, si nous intégrons dans nos calculs de la corésidence paroissiale tous les cas de cohabitation familiale d'enfants mariés et de parents mariés ou de veufs, la situation se renverse en faveur des fils $(0,28$ fils $/ 0,22$ gendres). La cohabitation familiale influe de la même manière sur les données concernant la corésidence des parents de l'époux et de l'épouse. La présence du père ou de la mère du chef de ménage est légèrement plus faible que celle des beaux-parents dans les données qui concernent strictement les liens de parenté entre les chefs de ménage $(0,15$ parents $/ 0,18$ beaux-parents). Toutefois, la proximité résidentielle du père ou de la mère de l'époux devance celle de ses beaux-parents, si nous intégrons encore dans nos calculs sur la corésidence familiale les données relatives aux couples, aux veufs et aux veuves vivant en cohabitation familiale $(0,26$ parents / 0,21 beaux-parents). Nos données indiquent une faible supériorité du modèle résidentiel virilocal et cette supériorité est plus nette dans le groupe paysan où la cohabitation familiale de l'un des garçons avec ses vieux parents découle directement du système de transmission des avoirs familiaux.

Ces écarts de proximité résidentielle selon le genre entre Lavaltrie et le Saguenay sont sans doute en grande partie liés au degré d'ancienneté des terroirs. Conformément au modèle de reproduction sociale décrit par G. Bouchard, les familles saguenayennes peuvent davantage concrétiser une stratégie d'établissement du plus grand nombre de fils possibles dans l'agriculture, à proximité des parents, compte tenu des terres disponibles et des possibilités de redéploiement dans l'espace régional. Par ailleurs, l'approche respective des deux recherches et la dimension très inégale des territoires étudiés influent sans doute quelque peu sur les résultats. Dans notre étude, nous mesurons la présence effective des enfants ou des parents, à un moment précis, dans une paroisse donnée, tandis que la démar- 
che de G. Bouchard consiste à suivre ces mêmes acteurs sur plusieurs décennies, dans leur déplacement d'une paroisse à l'autre, à l'échelle d'une région. Certes, nous n’avons pas la prétention de présenter une méthode plus adéquate pour saisir cette dimension de la dynamique familiale, mais notre approche devrait rendre plus facile la cueillette de données comparatives sur cette question.

\section{LES RÉSEAUX DE PARENTÉ ET}

\section{LES GROUPES SOCIOPROFESSIONNELS}

L'étendue des réseaux de parenté varie d'un ménage à l'autre. Nous voulons plus précisément vérifier si l'importance des réseaux de parenté à l'intérieur même de la paroisse fluctue selon le statut social du chef de ménage. Nous calculons donc le nombre moyen de liens de parenté des chefs de ménage de Lavaltrie, selon les groupes socioprofessionnels, en utilisant les déclarations de métiers et de statuts dans le recensement (tableau 4).

TABLEAU 4

Nombre moyen de liens de parenté proche et de parenté élargie des chefs de ménage de Lavaltrie selon leur statut socioprofessionnel, 1861

\begin{tabular}{l|c|c|c}
\hline $\begin{array}{l}\text { Statut socioprofessionnel } \\
\text { des chefs de ménage }\end{array}$ & $\begin{array}{c}\text { Nombre } \\
\text { de cas }\end{array}$ & $\begin{array}{c}\text { Nombre moyen } \\
\text { de liens de } \\
\text { parenté proche }\end{array}$ & $\begin{array}{c}\text { Nombre moyen } \\
\text { de liens de } \\
\text { parenté élargie }\end{array}$ \\
\hline Curé & 1 & 0 & 0 \\
Médecin & 1 & 0 & 0 \\
Marchand & 3 & 2,7 & 6,0 \\
Hôtelier & 1 & 1,0 & 11,0 \\
Bourgeois & 3 & 2,7 & 19,0 \\
Bedeau & 1 & 2,0 & 8,0 \\
Artisan & 16 & 2,2 & 9,3 \\
Cultivateur & 122 & 3,3 & 13,2 \\
Journalier & 43 & 2,3 & 7,8 \\
Mendiant & 1 & 2,0 & 12,0 \\
Rentier & 2 & 2,0 & 8,0 \\
Statut indéterminé & 3 & 1,0 & 8,3 \\
\hline Total & 197 & 2,9 & 11,4 \\
\hline
\end{tabular}


Le nombre de chefs de ménage dont la profession suggère la notabilité est peu élevé. Nous pouvons néanmoins constater l'absence les liens familiaux dans la communauté du curé et du médecin de la paroisse et le nombre plutôt faible de liens de parenté des marchands. Le curé, le médecin et l'un de ces marchands, Isaac Benoit, sont originaires de l'extérieur de la paroisse. Ce dernier, un immigrant français, a toutefois noué des relations plus étroites avec les familles de Lavaltrie en épousant en deuxièmes et en troisièmes noces des filles du terroir. Son fils, également marchand dans le village, a pour sa part épousé une fille de la ville de Montréal. Le dernier marchand, François Brault, est davantage intégré aux réseaux de parenté locaux puisque lui et sa conjointe, Elmire Brault, sont issus de vieilles familles paysannes de Lavaltrie.

D'autres déclarations de profession ou de statut, comme hôtelier et bourgeois, suggèrent un rang social proche de la notabilité. Les chefs de ménage de ce second groupe sont davantage intégrés dans les réseaux familiaux de Lavaltrie. L'hôtelier, Albert Lafontaine, originaire de l'extérieur de la paroisse, ne compte cependant qu'un seul parent proche dans la paroisse, le grand-père de sa conjointe, et la plupart de ses autres parents de Lavaltrie sont des oncles ou des cousins de celle-ci. Le profil des bourgeois est fort différent de celui de l'hôtelier. Ces anciens cultivateurs de la paroisse, dont le titre de bourgeois constitue une sorte de reconnaissance sociale, ont un nombre moyen de liens de parenté proche légèrement inférieur à la moyenne des chefs de ménage, mais un nombre moyen de liens de parenté élargie nettement supérieur à cette moyenne.

Les cultivateurs forment à la fois le groupe socioprofessionnel le plus nombreux dans la paroisse et celui qui affiche, en moyenne, le plus de liens de parenté proche et de parenté élargie, exception faite des bourgeois, qui proviennent par ailleurs de la paysannerie. Plusieurs travaux récents ont à juste titre insisté sur la forte mobilité des familles paysannes dans le Québec préindustriel. À chaque génération, l'importance des excédents démographiques commandait en quelque sorte un mouvement continu de la population des terroirs plus anciens vers les terroirs en expansion pour assurer, entre autres, l'établissement du plus grand nombre possible d'enfants dans l'agriculture, à titre d'exploitants propriétaires. Cette mobilité géographique, liée au mode de reproduction sociale, s'effectuait souvent par grappes familiales ${ }^{55}$. Dans la deuxième moitié du $\mathrm{xIx}^{\mathrm{e}}$ siècle, la saturation des terres dans la vallée du Saint-laurent et l'industrialisation modifient 
les perspectives de cette mobilité, tant dans les destinations que dans les destins sociaux des migrants. Cependant, d'autres travaux montrent bien que les réseaux familiaux demeurent alors une dimension fondamentale du phénomène migratoire ${ }^{56}$.

Par contre, on connaît beaucoup moins bien les liens entre le statut socio-économique, la densité des réseaux familiaux et le processus d'enracinement d'une partie de la population dans un terroir. Les données sur les liens de parenté, à Lavaltrie, apportent un premier élément de réflexion. La densité des liens familiaux des cultivateurs, à l'intérieur de la communauté locale, est supérieure à celle des autres groupes socioprofessionnels, tant chez les notables que dans les autres catégories des classes populaires rurales, comme les artisans et les journaliers.

Les artisans représentent en quelque sorte un petit groupe de chefs de ménage qui, concernant la densité de leurs réseaux familiaux à l'intérieur même de la paroisse, se situe à un niveau intermédiaire au sein des classes populaires. Au bas de l'échelle sociale, les journaliers forment un groupe dont les assises familiales, à l'intérieur même de la paroisse, sont nettement plus faibles que celles des cultivateurs.

Ces derniers, malgré leur statut commun d'exploitants propriétaires, ne constituent pas un groupe social homogène. L'étendue, la capacité de production et la valeur des exploitations agricoles varient considérablement d'un ménage paysan à l'autre. Nous croyons donc nécessaire de vérifier l'impact de la différenciation économique, entre les cultivateurs, sur la densité des liens familiaux dans la paroisse. Nous avons donc calculé le nombre moyen de liens de parenté proche et de parenté élargie pour quatre catégories de cultivateurs ou de ménages paysans déterminées à partir de la valeur de leurs patrimoines fonciers (tableau 5).

Les quatre catégories de cultivateurs enregistrent en moyenne plus de liens de parenté dans la paroisse de Lavaltrie que les artisans, les journaliers ou les notables. Cependant, ces nombres moyens de liens sont moins élevés dans les deux catégories dont la valeur des propriétés se situe sous l'écart type, et plus spécifiquement dans la catégorie inférieure à la moitié de l'écart type. Par contre, les plus riches cultivateurs ne sont pas nécessairement ceux dont les réseaux de parenté, à l'intérieur de la paroisse, sont les plus denses puisque c'est la catégorie se situant entre la valeur de l'écart type et le double de cette valeur qui détient le nombre moyen le plus élevé de liens de parenté proche et de parenté élargie.

56. F. Gagnon, op. cit.; B. Ramirez, op. cit.; B. Ramirez et J. Lamarre, op. cit. 
TABLEAU 5

Nombre moyen de liens de parenté proche et de parenté élargie des cultivateurs selon la valeur de leurs terres, Lavaltrie 1861

\begin{tabular}{l|c|c|c}
\hline $\begin{array}{l}\text { Catégories de } \\
\text { cultivateurs selon la } \\
\text { valeur de leurs terres }\end{array}$ & $\begin{array}{c}\text { Nombre } \\
\text { de cas }\end{array}$ & $\begin{array}{c}\text { Nombre moyen } \\
\text { de liens de } \\
\text { parenté proche }\end{array}$ & $\begin{array}{c}\text { Nombre moyen } \\
\text { de liens de } \\
\text { parenté élargie }\end{array}$ \\
\hline Catégorie 1 (- de 1238\$) & 34 & 2,8 & 10,8 \\
Catégorie 2 (1238-2476\$) & 30 & 3,1 & 12,2 \\
Catégorie 3 (2476-4952\$) & 29 & 4,0 & 17,1 \\
Catégorie 4 (4952\$ et +) & 28 & 3,6 & 13,2 \\
\hline
\end{tabular}

Ces données sur les nombres moyens de liens de parenté font état d'écarts entre les groupes. Cependant, dans chaque groupe socioprofessionnel et dans chacune des catégories de cultivateurs, le nombre de liens de parenté varie d'un ménage à l'autre. Nous avons vérifié les variations de liens de parenté élargie dans les principaux sous-groupes identifiés aux classes populaires rurales : les journaliers, les artisans et les quatre catégories de cultivateurs selon la valeur de leurs patrimoines fonciers (tableau 6).

Certes, le nombre de liens de parenté élargie d'un chef de ménage, à l'intérieur de la paroisse, n'est pas nécessairement lié à son statut social. Cependant, l'appartenance à un groupe spécifique influe assez généralement sur l'importance des réseaux familiaux détectés à l'intérieur de la paroisse. À Lavaltrie, en 1861, la moyenne des liens de parenté élargie d’un

TABLEAU 6

La distribution des chefs de ménage des classes populaires rurales selon le nombre de leurs liens de parenté élargie, Lavaltrie, 1861

\begin{tabular}{l|c|c|r|r|r|r|r}
\hline & \multicolumn{7}{|c}{ Le nombre de liens de parenté } \\
\hline $\begin{array}{l}\text { Groupes sociaux } \\
\text { et sous-groupes }\end{array}$ & $\begin{array}{c}\text { Nombre } \\
\text { de cas }\end{array}$ & Aucun & $\mathbf{1 - 5}$ & $\mathbf{6 - 1 0}$ & $\mathbf{1 1 - 1 5}$ & $\mathbf{1 6 - 2 0}$ & $\mathbf{2 1}$ et + \\
\hline Journaliers & 43 & 2 & 16 & 14 & 7 & 3 & 1 \\
Artisans & 16 & 1 & 5 & 5 & 2 & 2 & 1 \\
Catégorie 1 (- de 1238\$) & 34 & 0 & 14 & 5 & 4 & 6 & 5 \\
Catégorie 2 (1238-2476\$) & 30 & 1 & 10 & 4 & 4 & 1 & 10 \\
Catégorie 3 (2476-4952\$) & 29 & 0 & 4 & 1 & 7 & 6 & 11 \\
Catégorie 4 (4952\$ et +) & 29 & 0 & 7 & 8 & 4 & 4 & 6 \\
\hline
\end{tabular}


chef de ménage, à l'intérieur de la paroisse, est de 11,4. Or, environ $75 \%$ des journaliers et près de $69 \%$ des artisans enregistrent 10 liens et moins de parenté élargie. Dans ces deux groupes, les chefs de ménage disposant de plus de 20 liens de parenté constituent des exceptions. Dans les deux catégories inférieures de cultivateurs, selon la valeur des patrimoines fonciers, environ la moitié des chefs de ménage a 10 liens et moins de parenté élargie. Les cultivateurs les moins nantis, de la catégorie 1, se situent un peu plus fréquemment sous les 10 liens et, surtout, nettement moins souvent au-dessus de 20 liens que les cultivateurs de la catégorie 2 . Cette dernière catégorie est par ailleurs la plus polarisée. La moitié des chefs de ménage de cette catégorie compte 10 liens et moins de parenté, mais le tiers plus de 20 liens. Par contre, la plupart des cultivateurs de la catégorie 3 disposent d'une bonne assise familiale dans la paroisse puisque seulement $17 \%$ d'entre eux ont 10 liens et moins de parenté élargie. Ce groupe jouit également de la proportion la plus importante de chefs de ménage comptant plus de 20 liens de parenté, soit $37 \%$. La majorité des cultivateurs de la catégorie 4 ne dispose pas de réseaux familiaux aussi denses dans le milieu local. Plus de $52 \%$ d'entre eux ont 10 liens et moins de parenté à Lavaltrie tandis que seulement $21 \%$ cumulent plus de 20 liens.

De manière générale, les artisans et les journaliers reposent sur des assises familiales dans la paroisse moins étendues que celles des cultivateurs. Pour ces derniers, la valeur des exploitations représente un facteur pouvant favoriser l'enracinement des individus et des familles. Par contre, à l'échelle supérieure de la paysannerie, la possession de riches patrimoines fonciers peut entraîner le déploiement de l'espace familial au-delà des limites paroissiales de manière à participer plus adéquatement au processus d'accumulation de terres.

\section{LA DYNAMIQUE DES LIENS DE PARENTÉ ENTRE LES GROUPES}

Jusqu'à présent, nous avons constaté des différences dans l'étendue des liens de parenté selon le statut socioprofessionnel et, à l'intérieur de la paysannerie, selon l'importance du patrimoine foncier. Certes, le nombre de ces liens varie également au sein de chacun des groupes. Cependant, la différenciation sociale demeure fortement présente dans la distribution des chefs de ménage selon le nombre de liens de parenté dans la paroisse.

Cette différenciation sociale ne se vérifie pas seulement dans l'ampleur relative des réseaux de parenté à l'intérieur de la paroisse. Nous pouvons également constater la distance sociale entre les diverses strates des classes populaires rurales en étudiant, pour chacune de ces strates, la distribution 
sociale de la parenté proche et de la parenté élargie. Nous avons alors établi pour les principaux groupes concernés la ventilation sociale de ces deux niveaux de parenté (tableaux $7 \mathrm{a}$ et $7 \mathrm{~b}$, voir aussi les deux figures annexées).

TABLEAU 7 a

Ventilation sociale de la parenté proche des chefs de ménage de Lavaltrie, 1861 (en \%)

\begin{tabular}{l|c|r|r|r|r|r|r|c}
\hline & \multicolumn{5}{|c|}{$\begin{array}{c}\text { Pourcentage des parents proches selon } \\
\text { le statut du chef de ménage }\end{array}$} & \multirow{2}{*}{$\begin{array}{c}\text { Total } \\
\%\end{array}$} \\
\hline \multicolumn{1}{c|}{ Groupes } & Journalier & Cult. 1 & Cult. 2 & Cult. 3 & Cult. 4 & Artisan & Autres & A \\
\hline Journalier & 48,4 & 22,6 & 6,2 & 5,1 & 5,1 & 7,2 & 5,4 & 100,0 \\
Cult. 1 & 23,4 & 14,1 & 22,8 & 22,8 & 5,4 & 3,3 & 8,2 & 100,0 \\
Cult. 2 & 8,7 & 23,9 & 34,7 & 15,2 & 8,7 & 4,3 & 4,5 & 100,0 \\
Cult. 3 & 4,3 & 18,2 & 14,8 & 25,2 & 22,6 & 5,2 & 9,7 & 100,0 \\
Cult. 4 & 4,9 & 11,9 & 7,9 & 32,7 & 31,7 & 5,0 & 5,9 & 100,0 \\
Artisan & 20,0 & 11,5 & 11,5 & 22,7 & 11,5 & 17,1 & 5,7 & 100,0 \\
Autres & 10,7 & 14,3 & 10,7 & 14,3 & 21,4 & 10,7 & 17,9 & 100,0 \\
\hline
\end{tabular}

TABLEAU 7 b

Ventilation sociale de la parenté élargie des chefs de ménage de Lavaltrie, 1861 (en \%)

\begin{tabular}{l|c|c|c|c|c|c|c|c}
\hline & \multicolumn{5}{|c|}{$\begin{array}{c}\text { Pourcentage des parents proches selon } \\
\text { le statut du chef de ménage }\end{array}$} & \multirow{2}{*}{$\begin{array}{c}\text { Total } \\
\%\end{array}$} \\
\hline \multicolumn{1}{c|}{ Groupes } & Journalier & Cult. 1 & Cult. 2 & Cult. 3 & Cult. 4 & Artisan & Autres & Journalier \\
Jult. 1 & 33,4 & 18,8 & 13,7 & 10,8 & 10,2 & 7,2 & 5,9 & 100,0 \\
Cult. 2 & 18,5 & 19,6 & 19,6 & 18,5 & 11,5 & 8,7 & 3,6 & 100,0 \\
Cult. 3 & 13,4 & 21,5 & 21,8 & 20,7 & 12,5 & 4,1 & 6,0 & 100,0 \\
Cult. 4 & 8,5 & 14,7 & 15,3 & 28,0 & 20,2 & 4,6 & 8,7 & 100,0 \\
Artisan & 10,5 & 13,8 & 14,1 & 30,0 & 19,7 & 4,3 & 7,6 & 100,0 \\
Autres & 23,7 & 23,0 & 9,4 & 19,6 & 12,8 & 9,4 & 2,1 & 100,0 \\
\hline
\end{tabular}




\section{GRA PHI Q U E}

\section{Ventilation sociale des liens de parenté proche des chefs de ménage de Lavaltrie en 1861}

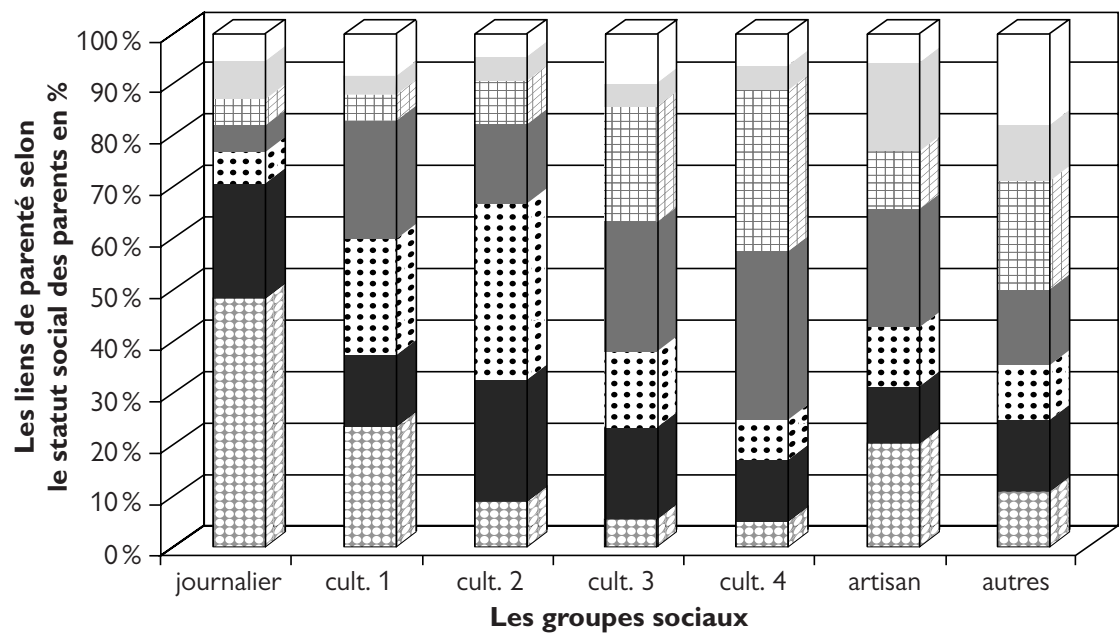

GRAPHIQUE 2

Ventilation sociale des liens de parenté élargie des chefs de ménage de Lavaltrie en 1861

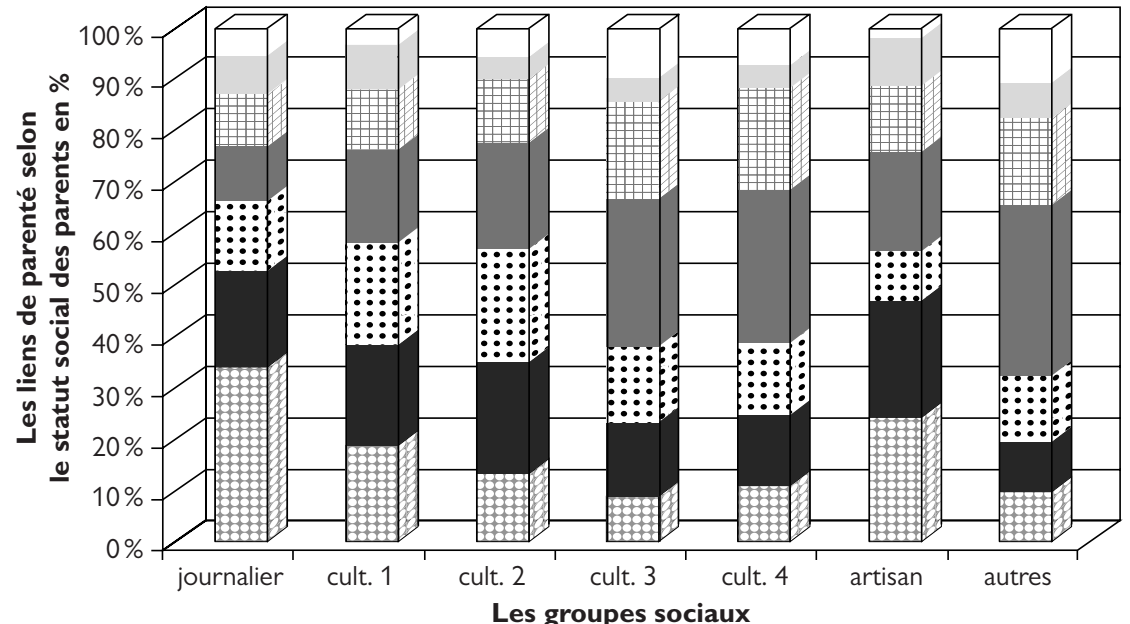

田 journalier $\square$ cult. $1 \square$ cult. $2 \square$ cult. 3

四 cult. $4 \quad \square$ artisan $\square$ autres 
Les réseaux familiaux ne sont pas totalement fermés ou repliés sur un groupe social spécifique. Ces réseaux réunissent, dans un même cercle, des chefs de ménage issus des divers groupes socioprofessionnels et de diverses catégories de cultivateurs. Néanmoins, la structure des réseaux de parenté demeure, du point de vue social, fortement préférentielle.

$\mathrm{Au}$ bas de l'échelle sociale, les journaliers tirent près de la moitié de leurs parents proches de leurs semblables. Un autre cinquième de leur parenté proche provient de la catégorie inférieure des cultivateurs telle que déterminée à partir de la valeur des patrimoines fonciers. Les journaliers ont par ailleurs peu de parents proches parmi les cultivateurs des deux catégories supérieures. Leur parenté élargie pénètre davantage ces deux groupes, mais cette parenté plus large demeure majoritairement confinée aux strates inférieures de la société rurale.

Les artisans, relativement peu nombreux dans la paroisse, sont davantage ouverts aux autres groupes socioprofessionnels. Seulement $17 \%$ de leur parenté proche et moins de $10 \%$ de leur parenté élargie proviennent du milieu artisanal en tant que tel. Les réseaux familiaux des artisans pénètrent autant les journaliers et les cultivateurs moins nantis que les cultivateurs plus fortunés. La structure de leurs réseaux familiaux, ouverte et relativement éclatée, fait état d'un groupe social dont la cohésion n'est pas poussée en milieu rural ${ }^{57}$.

Les réseaux familiaux des cultivateurs de la catégorie 1 sont également diversifiés. Ce groupe fournit lui-même une proportion relativement faible de sa parenté proche et de sa parenté élargie, compte tenu de son importance numérique parmi l'ensemble des cultivateurs. Cependant, l'ouverture vers les autres groupes s'effectue de manière un peu plus étroite que chez les artisans. Les liens de parenté entre cette catégorie de cultivateurs et les journaliers sont passablement importants : environ le cinquième de l'ensemble des liens. Leurs réseaux familiaux se dirigent aussi vers les catégories supérieures, mais ils pénètrent très faiblement la catégorie des cultivateurs possédant les plus riches patrimoines fonciers. Les cultivateurs

57. Dans une étude sur les charpentiers et les menuisiers de la région de Montréal au XviII ${ }^{\mathrm{e}}$ siècle, F.-L. Langlois constate une cohésion sociale beaucoup moins forte de ces artisans à la campagne qu'à la ville. Voir F.-L. Langlois, Les charpentiers et les menuisiers de la plaine de Montréal au 18 e siècle, mémoire de maîtrise (histoire), Université de Montréal, 1997. Pour le début du XIX ${ }^{\mathrm{e}}$ siècle, l'étude de Joanne Burgess sur les métiers du cuir à Montréal révèle une forte cohésion familiale et sociale des artisans de ce secteur dans le milieu urbain. Voir J. Burgess, Work, Family and Community: Montreal Leather Craftsmen, 1790-1831, thèse de doctorat (histoire), Université du Québec à Montréal, 1986. 
de la catégorie 1 recensent près de $23 \%$ de leur parenté proche dans chacune des deux catégories subséquentes de cultivateurs contre environ $5 \%$ seulement dans la strate supérieure des cultivateurs dont le nombre de chefs de ménage est pourtant sensiblement similaire à chacune des deux autres catégories. Le pourcentage de leur parenté élargie issue des cultivateurs de la catégorie 4 est deux fois plus important, à plus de $11 \%$. Cependant, ces gains vers le haut se font surtout aux dépens des cultivateurs de la catégorie 3.

Les cultivateurs de la catégorie 2 puisent une proportion beaucoup plus forte de leurs parents proches dans leur groupe que ceux de la catégorie 1 , soit plus du tiers. Une partie importante de cette parenté proche, près de $40 \%$, provient par ailleurs des deux catégories de cultivateurs immédiatement au-dessous et au-dessus dans la hiérarchie des avoirs fonciers. Ces cultivateurs de la catégorie 2 ont moins de liens de parenté dans le bas de l'échelle sociale, parmi les journaliers, que ceux de la catégorie 1. Par ailleurs, la structure de la parenté élargie de cette deuxième catégorie de cultivateurs ressemble beaucoup à celle de la première catégorie. Notons toutefois une part moins importante des journaliers (13\% contre $18 \%$ ) et des artisans ( $4 \%$ par rapport à près de $9 \%$ ).

Les réseaux de parenté proche et de parenté élargie des cultivateurs de la catégorie 3 tendent leurs ramifications vers les échelons supérieurs des classes populaires rurales. La place des artisans demeure relativement marginale, à environ $5 \%$ dans les deux types de parenté, tandis que celle des journaliers chute à moins de $5 \%$ de la parenté proche et à seulement $8,5 \%$ de la parenté élargie. Les cultivateurs de la catégorie 3 concentrent par ailleurs environ $30 \%$ de leur parenté proche et élargie dans les deux catégories inférieures de cultivateurs et environ $50 \%$ dans les deux catégories supérieures. La ventilation sociale des réseaux de parenté des cultivateurs de la catégorie 4 confirme la tendance décrite chez les cultivateurs de la catégorie 3. Ces propriétaires des plus riches patrimoines fonciers de la paroisse limitent davantage leur parenté aux membres des deux catégories supérieures de cultivateurs déterminées selon la valeur des terres.

\section{CONCLUSION}

Jusqu'à présent, la littérature scientifique a beaucoup insisté sur l'importance de la parenté et des réseaux familiaux dans la société rurale du Québec préindustriel. Cette étude de cas n’a pas la prétention de réfuter ce constat. Elle permet toutefois de nuancer la densité relative des liens de parenté et l'étendue des réseaux familiaux dans une vieille paroisse rurale 
de la vallée du Saint-Laurent au milieu du $\mathrm{xix}^{\mathrm{e}}$ siècle : Saint-Antoine-deLavaltrie. Il manque apparemment certaines mailles au fameux tricot familial. Néanmoins, il faudra de nombreuses études comparatives, dans le temps et dans l'espace, avant de mieux déterminer l'importance réelle de ces réseaux.

Dans cette paroisse, la plupart de chefs de ménage et leurs conjointes comptent un noyau plutôt réduit de parents proches parmi l'ensemble des chefs de ménage de la même paroisse, tandis que les réseaux de parenté plus large, jusqu'au cousinage, réunissent en moyenne, dans un même noyau de parents, seulement 5 à $6 \%$ des ménages de la paroisse. De plus, l'étendue de ces réseaux familiaux est très inégale d'un ménage à l'autre, variant fortement selon le statut social des chefs de ménage. Comme C. Collard l'avait déjà constaté pour une paroisse rurale de Charlevoix au XIx ${ }^{\mathrm{e}}$ siècle, nous notons une nette distinction des réseaux de parenté selon le statut social. Les cultivateurs, déjà bien enracinés à leur terroir par le biais de la propriété foncière, constituent aussi le groupe social bénéficiant du plus large réseau de parenté dans la paroisse, tandis qu'au bas de l'échelle sociale, les journaliers comptent généralement peu de parents dans la communauté locale. Par ailleurs, parmi les cultivateurs, l'étendue de ces réseaux de parenté varie assez fortement selon la valeur des patrimoines fonciers possédés. Cette différenciation ne concerne pas seulement l'étendue relative des réseaux de parenté; elle se vérifie également dans la distribution sociale de la parenté dans les diverses strates des classes populaires : artisans, journaliers, cultivateurs pauvres ou aisés. Certes, ces réseaux de parenté d'un groupe social spécifique englobent assez généralement des parents issus des autres groupes sociaux. Cette circulation des parents dans l'espace social suggère une certaine flexibilité des structures sociales et, surtout, une assez grande fluidité des rapports sociaux dans les campagnes. Néanmoins, la composition sociale des réseaux de parenté de chacun de ces groupes, surtout la parenté proche, demeure assez fortement sélective selon la place des familles dans la hiérarchie locale.

Cette étude apporte aussi des connaissances utiles sur la structure des ménages et des réseaux de parenté. Cette vieille paroisse rurale de la vallée du Saint-Laurent, du moins dans une perspective nord-américaine, compte une proportion importante de ménages complexes et semicomplexes. La place relative de ces deux types de ménages est en grande partie liée au système de reproduction sociale. Le mode de transmission préférentielle de la terre familiale d'un père à l'un de ses garçons y entraîne 
assez fréquemment la cohabitation du fils donataire et de sa conjointe avec ses deux parents (ménages complexes) et, après le décès de l'un des donateurs, avec le parent survivant (ménages semi-complexes). Cette prédominance des liens patrilinéaires dans la cohabitation concerne d'ailleurs plus fortement le milieu paysan, et surtout les catégories de cultivateurs possédant les plus riches patrimoines fonciers. Cependant, malgré ce biais agnatique dans le mode de transmission des biens et son impact sur la cohabitation familiale, nous n'avons pas constaté, contrairement au cas saguenayen, une nette prédominance du modèle résidentiel virilocal. À Saint-Antoine-de-Lavaltrie, les femmes et les liens matrilinéaires conservent une place assez importante dans le bilan global des réseaux de parenté à l'intérieur de la paroisse.

Enfin, ces nouvelles données sur la parenté n’infirment pas les conclusions des travaux antérieurs sur l'importance des réseaux familiaux dans les migrations ou dans les modalités de formation de la classe ouvrière. La fréquence des liens familiaux entre les migrants d'une même destination ou entre les travailleurs d'un même métier et d'une même entreprise devient, selon nous, d'autant plus significative que, dans les communautés locales d'origine, la densité réelle des réseaux familiaux ne peut pas entraîner de manière fortuite ces regroupements de parents. Dans la même perspective, la constatation de nombreux liens familiaux entre des élites institutionnelles locales n'est pas un exercice futile. Cette démarche permet effectivement de dévoiler une certaine forme de concentration du pouvoir dans ces communautés. L'étude des réseaux de parenté dans le fonctionnement de la société demeure un champ de recherche plein de promesses. 